MATHEMATICS OF COMPUTATION

Volume 68, Number 225, January 1999, Pages 91-122

S $0025-5718(99) 01044-3$

\title{
ON THE EXTRACTION TECHNIQUE IN BOUNDARY INTEGRAL EQUATIONS
}

\author{
C. SCHWAB AND W. L. WENDLAND
}

This work is dedicated to Professor Dr. G. C. Hsiao on the occasion of his $60^{\text {th }}$ birthday

\begin{abstract}
In this paper we develop and analyze a bootstrapping algorithm for the extraction of potentials and arbitrary derivatives of the Cauchy data of regular three-dimensional second order elliptic boundary value problems in connection with corresponding boundary integral equations. The method rests on the derivatives of the generalized Green's representation formula, which are expressed in terms of singular boundary integrals as Hadamard's finite parts. Their regularization, together with asymptotic pseudohomogeneous kernel expansions, yields a constructive method for obtaining generalized jump relations. These expansions are obtained via composition of Taylor expansions of the local surface representation, the density functions, differential operators and the fundamental solution of the original problem, together with the use of local polar coordinates in the parameter domain. For boundary integral equations obtained by the direct method, this method allows the recursive numerical extraction of potentials and their derivatives near and up to the boundary surface.
\end{abstract}

\section{INTRODUCTION}

The evaluation of layer potentials and also their derivatives near the supporting surface $\Gamma \subset \mathbb{R}^{d}, d=2$ or 3 , has attracted attention in boundary element computations recently (see e.g., $[8,10,11,12,20]$ ). If the point of interest $x$ is sufficiently distant from $\Gamma$, the evaluation does not cause any difficulties due to the smoothness of the kernels. In contrast, if $x$ is very close to $\Gamma$, severe numerical difficulties arise due to the oscillatory nature of the kernels, related to the so-called jump relations satisfied by the potentials at $\Gamma$. Much effort was spent on numerical techniques to cope with this problem. Such techniques have limited success if the analytic jump relations of the potentials are ignored. To illustrate this, consider the double layer potential of the density $\chi_{[-1,1]^{2}}(y)$ as a function of the observation point $x=(0,0, \varepsilon)$ :

$$
W \chi(x)=\int_{-1}^{1} \int_{-1}^{1} \frac{n(y) \cdot(x-y)}{|x-y|^{3}} d y_{1} d y_{2}=2 \pi \operatorname{sign} \varepsilon-8 \arcsin \left(\frac{\varepsilon \sqrt{2}}{2 \sqrt{1+\varepsilon^{2}}}\right) .
$$

Received by the editor April 29, 1997.

1991 Mathematics Subject Classification. Primary 45F15, 15N38, 45K05; Secondary 47G30, $58 \mathrm{G} 15,35 \mathrm{~J} 25$.

Key words and phrases. Boundary integral equation methods, derivatives of the Cauchy data, regularization of hypersingular potentials.

(C)1999 American Mathematical Society 


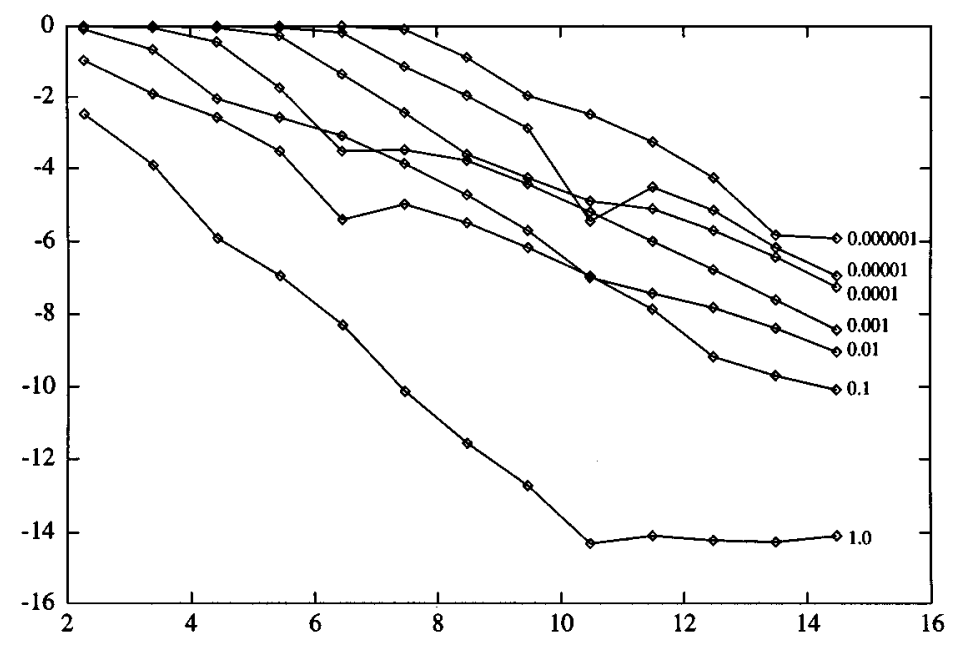

FiguRE 1. $\log _{10}$ (relative error) versus $N^{1 / 3}$

Figure 1 shows the relative quadrature error for $\varepsilon=10^{-j}, j=0, \ldots, 6$, of an advanced quadrature scheme applied to (1.1) (see [33] for details).

The degeneracy for small $\varepsilon$ is clearly visible and even more pronounced for less sophisticated quadrature schemes.

In this paper, we propose a different, analytical approach: Since the potentials used in the reduction to the boundary $\Gamma$ are analytic on either side of $\Gamma$ and admit analytic extensions through $\Gamma$ (provided $\Gamma$ and the data are analytic), they admit Taylor expansions about a point $x_{0} \in \Gamma$. To illustrate our approach, consider the classical potential problem

$$
\Delta U=0 \quad \text { in } \Omega^{c}, \quad \frac{\partial U}{\partial n}=\psi \quad \text { on } \Gamma, \quad U(x)=\mathcal{O}\left(\frac{1}{|x|}\right) \quad \text { for }|x| \rightarrow \infty .
$$

Here and in what follows, $\Omega \subset \mathbb{R}^{d}$ denotes a bounded, simply connected strong Lipschitz domain [30] with piecewise analytic boundary $\Gamma$. For $d=3, \Gamma$ is a finite union of analytic surface pieces $\bar{\Gamma}_{j}$, and it is locally the graph of a Lipschitzcontinuous function (precluding slits and cusps). For $d=2$, the $\bar{\Gamma}_{j}$ are analytic arcs, correspondingly. We denote by

$$
\widetilde{\Gamma}:=\bigcup \Gamma_{j}, S:=\Gamma \backslash \widetilde{\Gamma}
$$

the sets of smooth, i.e., analytic, and singular boundary points, respectively. For every $x \in \widetilde{\Gamma}, n_{x}=n(x)$ will denote the exterior unit normal vector. The complement $\mathbb{R}^{d} \backslash \bar{\Omega}$ of $\Omega$ will be denoted by $\Omega^{c}$. Then $n_{x}$ always points into $\Omega^{c}$.

The classical direct reduction method of (1.2) to the boundary $\Gamma$ is based on the representation formula

$$
U(x)=W U(x)-V \frac{\partial U}{\partial n} \text { for } x \in \Omega^{c} .
$$

Here $V, W$ are the single and double layer potentials of classical potential theory, respectively. For $x \rightarrow x^{\prime} \in \widetilde{\Gamma},(1.3)$ and the jump relations yield the boundary 
integral equation

$$
A \varphi=\left(\frac{1}{2} I-K\right) \varphi=-V \psi \quad \text { at } x^{\prime} \in \Gamma
$$

for the unknown Cauchy datum $\varphi:=U_{\mid \Gamma}$. Here, for $d=3$,

$$
V \psi(x)=\frac{1}{4 \pi} \int_{y \in \Gamma} \frac{\psi(y)}{|x-y|} d s_{y} \quad \text { for } x \in \Gamma
$$

is the single layer potential operator and

$$
K \varphi=\frac{1}{4 \pi} \int_{y \in \Gamma} \frac{(x-y) \cdot n_{y}}{|x-y|^{3}} \varphi(y) d s_{y} \quad \text { for } x \in \widetilde{\Gamma}
$$

is the double layer potential at the points of $\widetilde{\Gamma}$, which is to be modified appropriately at $x \in S$.

Once we solve the classical boundary integral equation (1.4) for $\varphi$, the Cauchy data $\varphi$ and $\psi$ are inserted into (1.3) and $U(x)$ is known for all $x \in \Omega^{c}$. Moreover, if $\psi$ is analytic on $\widetilde{\Gamma}$, so is $\varphi$ (see e.g., [29]) and, hence, also $U(x)$ at $x \in \Omega^{c} \cup \widetilde{\Gamma}$. Therefore, for $x$ sufficiently close to $\widetilde{\Gamma}$, there exists the nearest point $x_{0} \in \widetilde{\Gamma}$ and we may write

$$
U(x)=U\left(x_{0}\right)+\left|x-x_{0}\right| \frac{\partial U}{\partial n}+\frac{1}{2}\left|x-x_{0}\right|^{2} \frac{\partial^{2} U}{\partial n^{2}}\left(x_{0}\right)+\mathcal{O}\left(\left|x-x_{0}\right|^{3}\right) .
$$

The Taylor series corresponding to (1.7) converges for $x$ sufficiently close to $x_{0}$ in case of analytic data due to the analyticity of $U(x)$, and represents the solution $U$ for $x \in \Omega^{c} \cup \widetilde{\Gamma}^{1}{ }^{1}$ Using (1.7) yields approximations to $U(x)$ which improve as $x \rightarrow x_{0}$, in contrast to numerical evaluations of the representation formula (1.2).

The difficulty in using (1.7) is how to get $\frac{\partial^{k} U}{\partial n^{k}}\left(x_{0}\right)$ for $k \geq 1$. In our specific problem (1.2), the boundary datum $\frac{\partial U}{\partial n}\left(x_{0}\right)=\psi\left(x_{0}\right)$ is available, but not the higher order derivatives. To provide a stable algorithm by finding such derivatives from numerical solutions of the integral equation (1.4) is the purpose of the present paper.

Let us explain our approach for the special boundary value problem (1.2) for $d=3$. To this end, we need some standard background from the calculus of surfaces.

For the two-dimensional case $d=2$ the whole approach can rather easily be specified, correspondingly.

We call an open surface piece $\Gamma_{j} \subset \mathbb{R}^{3}$ analytic if it has a real analytic parametric representation $\chi_{j}: \mathbb{R}^{2} \rightarrow \mathbb{R}^{3}$ of

$$
\Gamma_{j}=\chi_{j}\left(V_{j}\right),
$$

with a bounded, polygonal parameter domain $V_{j} \subset \mathbb{R}^{2}$, and $\chi_{j}$ is analytic on $\bar{V}_{j}$, i.e., it does not exhibit singularities on $\partial V_{j}$. Hence $\chi_{j}$ can be extended analytically to some open neighborhood of $\bar{V}_{j}$ in $\mathbb{R}^{2}$. Throughout, $x, y$ will denote points in $\mathbb{R}^{d}$

\footnotetext{
${ }^{1}$ Note carefully that the Taylor series about $x_{0}$ allows us to extend $U$ to certain points $x \in \Omega$. However, this extension does not coincide with the solution of (1.2) in $\Omega$, nor with (1.3) for $x \in \Omega$.
} 
for $d=3$, i.e., $x=\left(x_{1}, x_{2}, x_{3}\right)^{\top}$ etc., and for $x, y \in \Gamma_{j}$ their local coordinates will be denoted by $u$ and $v$, respectively, i.e.,

$$
x=\chi_{j}\left(u_{1}, u_{2}\right) \in \Gamma_{j}, y=\chi_{j}\left(v_{1}, v_{2}\right) \in \Gamma_{j} .
$$

If no ambiguity arises or if we prove statements that do not depend on the particular surface piece, we omit the subscript $j$. Partial derivatives with respect to local coordinates will be written in tensor notation, i.e.,

$$
\frac{\partial \chi}{\partial v_{\nu}}=\chi_{\mid \nu}, \quad \frac{\partial^{2} \chi}{\partial v_{\nu} \partial v_{\varrho}}=\chi_{\mid \nu \varrho}, \quad \text { etc., } \quad \nu, \varrho=1,2 .
$$

We will assume that $\chi_{j}$ is regular, i.e.,

$$
\left|\chi_{j \mid 1} \times \chi_{j \mid 2}\right| \neq 0 \quad \text { for all } u \in \bar{V}_{j}
$$

and the mapping $\chi_{j}: V_{j} \rightarrow \Gamma_{j}$ is invertible. Then, for $y \in \Gamma_{j}$, the surface measure is given by

$$
d s_{y}=\sqrt{\gamma} d v \quad \text { with } \gamma=\left|\chi_{\mid 1}\right|^{2}\left|\chi_{\mid 2}\right|^{2}-\left(\chi_{\mid 1} \cdot \chi_{\mid 2}\right)^{2}
$$

and the metric tensor is $\gamma_{\nu \varrho}=\chi_{\mid \nu} \cdot \chi_{\mid \varrho}$ and its inverse $\left(\left(\gamma^{\iota \nu}\right)\right)=\left(\left(\gamma_{\nu \varrho}\right)\right)^{-1}$.

The exterior unit normal vector to $\Gamma$ at $x \in \Gamma_{j}$ is given by

$$
n(x):=\left|\chi_{j \mid 1} \times \chi_{j \mid 2}\right|^{-1}\left(\chi_{j \mid 1} \times \chi_{j \mid 2}\right) .
$$

We now construct an extension of $\chi_{j}$ so that a whole tubular neighborhood of $\Gamma_{j}$ in $\mathbb{R}^{3}$ is mapped bijectively to the cylinder $V_{j} \times(-\varepsilon, \varepsilon)$ with appropriately chosen $\varepsilon>0$.

Proposition 1.1. For every $\Gamma_{j} \subset \Gamma$ there exists $\varepsilon_{j}>0$ such that the open set

$$
\mathcal{U}\left(\Gamma_{j}\right):=\left\{y \in \mathbb{R}^{3}\left|y=\chi_{j}\left(v_{1}, v_{2}\right)+v_{3} n\left(v_{1}, v_{2}\right),\left(v_{1}, v_{2}\right) \in V_{j} \subset \mathbb{R}^{2},\right| v_{3} \mid<\varepsilon_{j}\right\}
$$

is diffeomorphic to

$$
\mathcal{V}\left(V_{j}\right):=\left\{v \in \mathbb{R}^{3}\left|\left(v_{1}, v_{2}\right) \in V_{j},\right| v_{3} \mid<\varepsilon_{j}\right\} .
$$

The diffeomorphism

$$
\Phi_{j}: \mathcal{V}\left(V_{j}\right) \rightarrow \mathcal{U}\left(\Gamma_{j}\right)
$$

in (1.13) is analytic, bijective, and extends $\chi_{j}$ in the sense that $\left.\Phi_{j}\right|_{v_{3}=0}=\chi_{j}\left(v_{1}, v_{2}\right)$. Moreover, $\Phi_{j}$ is regular on $\mathcal{V}\left(V_{j}\right)$.

Proof. We omit the subscript $j$ in this proof. Since the Jacobian of $\Phi$ at $v \in \mathcal{V}$ is

$$
|D \Phi(v)|=\gamma(v)\left(1-2 v_{3} H\left(v_{1}, v_{2}\right)+v_{3}^{2} G\left(v_{1}, v_{2}\right)\right)^{2},
$$

where $H$ and $G$ are the mean and the Gaussian curvatures, respectively, by the implicit function theorem there exists $0<\varepsilon_{0}=\min _{x \in \bar{\Gamma}_{j}}\left\{H-\sqrt{H^{2}-G}\right\} /|G|$ such that $\Phi$ is bijective, analytic, and extends $\chi$ for $\left|v_{3}\right|<\varepsilon \leq \varepsilon_{0}$.

The main tool in deriving formulae for the normal derivatives $\frac{\partial^{k} U}{\partial n^{k}}\left(x_{0}\right)$ is to express them by tangential derivatives of $\psi$ and $\varphi$ by using the differential equation $\Delta U=0$ in $\Omega^{c} \cup \widetilde{\Gamma}$. For instance, in the tubular neighbourhood $\mathcal{U}\left(\Gamma_{j}\right)$, the Laplace equation reads

$$
\Delta U\left(x_{0}\right)=\frac{\partial^{2} U}{\partial n^{2}}\left(x_{0}\right)-2 H\left(x_{0}\right) \frac{\partial U}{\partial n}\left(x_{0}\right)+\Delta_{\Gamma} U\left(x_{0}\right)=0,
$$


where

$$
\Delta_{\Gamma} U=\frac{1}{\sqrt{\gamma}} \sum \frac{\partial}{\partial v_{\iota}}\left(\sqrt{\gamma} \gamma^{\iota \nu} \frac{\partial}{\partial v_{\nu}}\right)(U \circ \chi(v))
$$

denotes the Laplace-Beltrami operator on $\Gamma$. This yields, in our case,

$$
\frac{\partial^{2} U}{\partial n^{2}}\left(x_{0}\right)=2 H\left(x_{0}\right) \psi\left(x_{0}\right)-\Delta_{\Gamma} \varphi\left(x_{0}\right) .
$$

Thus, since $\varphi$ is known as the solution of (1.4), its derivatives could be obtained directly and the Taylor formula (1.7) is, in principle, available.

In practical computations, however, (1.4) can only be solved by some approximation method like, e.g., boundary elements, and differentiation of the approximate solution $\varphi_{\text {appr }}$ is infeasible due to loss of accuracy (more precisely, derivatives of $\varphi_{\text {appr }}$ converge at a reduced rate; the reduction being proportional to the order of the derivative). Therefore, the viability of the approach depends on our ability to compute numerical approximations of tangential derivatives $\partial^{\nu} \varphi=\varphi_{\left.\right|_{\nu}}$ of $\varphi$ which exhibit the same accuracy as $\varphi_{\text {appr }}$. To this end, we derive a hierarchy of boundary integral equations for $\varphi_{\mid \nu}$ by differentiating (1.4). This yields for $|\nu|=1$ :

$$
A \varphi_{\mid \nu}=-V \psi_{\mid \nu}-A_{(\nu)} \varphi-V_{(\nu)} \psi
$$

where

$$
\begin{aligned}
& A_{(\nu)}=\left[\partial^{\nu}, A\right]:=\partial^{\nu} A-A \partial^{\nu}, \\
& V_{(\nu)}=\left[\partial^{\nu}, V\right]:=\left(\partial^{\nu} V-V \partial^{\nu}\right)
\end{aligned}
$$

are the commutators. Note that (1.17) is an integral equation for $\varphi_{\mid \nu}$ with the same operator $A$ as in (1.4) but with a modified right-hand side. The viability of this approach depends on the properties and computability of the commutators $A_{(\nu)}$ and $V_{(\nu)}$. Both commutators define pseudo-differential operators on $\Gamma_{j}$ of the same orders as $A$ and $V$ (here 0 and -1 ), respectively. In addition, we show that, in local coordinates, $A_{(\nu)}$ and $V_{(\nu)}$ can always be decomposed into commutators of differential operators whose kernels can be explicitly computed. For instance, if $d=3$, we have

$$
V_{(\nu)} \psi(x)=\frac{1}{4 \pi} \int_{\Gamma}\left\{\frac{(y-x) \cdot\left(\chi_{\mid \nu}(y)-\chi_{\mid \nu}(x)\right)}{|x-y|^{3}}+\frac{1}{|x-y|}(\log \sqrt{\gamma})_{\mid \nu}\right\} \psi(y) d s_{y}
$$

and $V_{(\nu)}$ is again a weakly singular integral operator. Relations analogous to (1.17) for $|\nu|>1$ are obtained in the same manner but with corresponding higher order commutators. For the second derivatives of $\varphi$, needed in (1.16), differentiation of (1.17) leads to the equation

$$
\begin{aligned}
A \varphi_{\mid \nu \mu}= & -V \psi_{\mid \nu \mu}-V_{(\mu)} \psi_{\mid \nu}-V_{(\nu)} \psi_{\mid \mu}-\left[\partial^{\mu}, V_{(\nu)}\right] \psi \\
& -A_{(\nu)} \varphi_{\mid \mu}-A_{(\mu)} \varphi_{\mid \nu}-\left[\partial^{\mu}, A_{(\nu)}\right] \varphi
\end{aligned}
$$

Note that the right-hand side is given in terms of derivatives of $\varphi$ not exceeding the order one and commutators of $A$ and $V$ the orders of which do not exceed the respective orders. Clearly, the datum $\psi$ in (1.20), i.e., in (1.2), needs to be given sufficiently smooth. The equations (1.4), (1.17) and (1.20) can be solved recursively for all the second derivatives $\varphi_{\left.\right|_{\nu \mu}}$. Numerical approximations of these derivatives can be computed provided the discretization of $A$ and its inversion is available and 
the commutators are known. For higher order derivatives, equations analogous to (1.20) will be obtained. In all cases, the operator on the left-hand side is $A$.

In the remainder of this paper we present the procedure for second order strongly elliptic systems generalizing (1.16), and also prove a general representation of the commutators in local coordinates which allows their explicit calculation.

Finally we address the rate of convergence of arbitrary derivatives, including the normal derivatives obtained from boundary element discretizations of the boundary integral equations like (1.4), (1.17) and (1.20).

The outline of the paper is as follows: In Section 2 we introduce the class of boundary value problems and reductions to the boundary integral equations to be considered. In Section 3 we derive the recurrence of boundary integral equations for arbitrary derivatives and give the formula for the calculation of the commutators. Finally, in Section 4 we address the boundary element discretization of the boundary integral equations for the higher derivatives of the solution, and obtain rates of convergence. An extraction technique for the computation of pointwise derivatives is also analyzed.

Although the method is analyzed here for smooth $\Gamma$, it can also be applied to piecewise smooth $\Gamma$ provided $x_{0}$ has an open neighbourhood where $\Gamma$ still is smooth. However, the commutator formula needs to be modified at corners and edges; we leave these modifications to future work.

The whole technique is presented for $d=3$. The corresponding two-dimensional case where $\Gamma$ consists of a closed curve can be derived from our presentation by corresponding modifications, and is presented in [32].

\section{Boundary VAlue PRoblems AND REDUCtions to THE BOUNDARY IN $\mathbb{R}^{3}$}

In this section we will discuss the solution of elliptic boundary value problems by equivalent boundary integral equations following the approach in [6] (see also [17], and for special cases [9]). For simplicity, we confine ourselves to elliptic systems of second order in variational form: Given $f \in L^{2}(\Omega)$, find $U \in H^{1}(\Omega)$ such that

$$
\mathcal{L} U=f \quad \text { in } \Omega .
$$

Here

$$
\mathcal{L}=-\sum_{j, k=1}^{3} D_{j} a_{j k} D_{k}+c
$$

$D_{j}=\frac{\partial}{\partial x_{j}}$, and the coefficients $a_{j k}$ and $c$ are real and analytic $N \times N$ matrix functions of $x \in \bar{\Omega}$ which satisfy

$$
a_{j k}(x)=a_{k j}^{\top}(x), \quad c(x)=c^{\top}(x)
$$

(i.e., $\mathcal{L}$ is formally selfadjoint). General boundary conditions, including Dirichlet and Neumann conditions, are as follows:

Let $P \in C^{\infty}\left(\Gamma, \mathbb{C}^{N \times N}\right)$ be a family of orthogonal projectors with constant rank, i.e., $P^{2}=P=\bar{P}^{\top}$. Then require the boundary conditions

$$
R\left(\gamma_{0} U, \gamma_{1} U\right):=P \gamma_{0} U+(I-P) \gamma_{1} U=\psi
$$


where, for $U \in C^{1}(\bar{\Omega})$,

$$
\gamma_{1} U=\left.\sum_{j, k=1}^{3} n_{j} a_{j k} D_{k} U\right|_{\tilde{\Gamma}}
$$

denotes the conormal derivative corresponding to $\mathcal{L}$. By $H^{k}\left(\Omega, \mathbb{C}^{N}\right)$ with $k \in \mathbb{N}_{0}$ we denote the usual Sobolev spaces. The spaces with noninteger $k>0$ are defined by interpolation (see for example [25]). The boundary spaces $H^{s}\left(\Gamma, \mathbb{C}^{N}\right)$ with $0<s<1$ are invariantly defined via a partition of unity on $\Gamma$ and the local Lipschitz maps describing $\Gamma$ [30]. For $-1<s<0$, we define $H^{s}\left(\Gamma, \mathbb{C}^{N}\right)$ via the $L^{2}$-duality on $\Gamma$. We further note that the trace operator $\gamma_{0}$ is continuous [4], i.e.,

$$
\gamma_{0}: H^{s}\left(\Omega, \mathbb{C}^{N}\right) \rightarrow H^{s-1 / 2}\left(\Gamma, \mathbb{C}^{N}\right) \quad \text { with } 1 / 2<s \leq 3 / 2 .
$$

We shall not indicate spaces of vector-valued functions explicitly when it will be clear from the context. Likewise, $H^{k}\left(\Omega, \mathbb{C}^{N}\right)$ will be denoted by $H^{k}(\Omega)$, etc.

For (2.1), (2.3) we have the first Green's formula

$$
\left\langle\gamma_{1} U, \gamma_{0} Y\right\rangle_{\Gamma}=B(U, Y)-\int_{\Omega} \bar{Y}^{\top} \mathcal{L} U d x \quad \text { with } Y \in H^{1}(\Omega), U \in H_{\mathcal{L}}(\Omega),
$$

where the sesquilinear form $B(\cdot, \cdot): H^{1}(\Omega) \times H^{1}(\Omega) \rightarrow \mathbb{C}$ is given by

$$
B(U, Y)=\int_{\Omega}\left\{\sum_{j, k=1}^{3} D_{j} \bar{Y}^{\top} a_{j k} D_{k} U+\bar{Y}^{\top} c U\right\} d x
$$

and

$$
H_{\mathcal{L}}(\Omega):=\left\{U \mid\|\mathcal{L} U\|_{L^{2}(\Omega)}<\infty\right\} \cap H^{1}(\Omega)
$$

equipped with the graph-norm $\|U\|_{H_{\mathcal{L}}(\Omega)}=\|U\|_{H^{1}(\Omega)}+\|\mathcal{L} U\|_{L^{2}(\Omega)}$.

The bracket $\langle\cdot, \cdot\rangle_{\Gamma}$ in $(2.6)$ is an extension of the boundary integral $\int_{\Gamma} \gamma_{1} U^{\top} \gamma_{0} \bar{Y} d s_{y}$ by continuity from smooth $U, Y$ to $U \in H_{\mathcal{L}}(\Omega), Y \in H^{1}(\Omega)$. Since the trace map is surjective, (2.6) defines $\gamma_{1} U$ for all $U \in H_{\mathcal{L}}(\Omega)$ as a continuous, linear functional on $H^{1 / 2}(\Gamma)$. Thus $\gamma_{1} U \in H^{-1 / 2}(\Gamma)$; and $\gamma_{1}: H_{\mathcal{L}}(\Omega) \rightarrow H^{-1 / 2}(\Gamma)$ is continuous. Analogously we extend the classical second Green's formula

$$
\int_{\Omega}\left\{\bar{Y}^{\top} \mathcal{L} U-U^{\top} \mathcal{L} \bar{Y}\right\} d x=\left\langle\gamma_{0} U, \gamma_{1} Y\right\rangle_{\Gamma}-\left\langle\gamma_{1} U, \gamma_{0} Y\right\rangle_{\Gamma}
$$

from $U, Y \in H^{2}(\Omega)$ to $U, Y \in H_{\mathcal{L}}(\Omega)$.

To give the weak formulation of the boundary value problems (2.3), we denote by $\mathcal{R}_{D}$ and $\mathcal{R}_{N}$ the finite-dimensional linear spaces of solutions to the corresponding homogeneous adjoint boundary value problem. Then the weak formulation of the boundary value problem (2.3) reads:

Given $\psi$ with $P \psi \in H^{1 / 2}(\Gamma)$ and $(I-P) \psi \in H^{-1 / 2}(\Gamma)$, and $f \in L^{2}(\Omega)$ satisfying

$$
\int \bar{r}^{\top} f d x=\left\langle P \psi, P \gamma_{1} r\right\rangle-\left\langle(1-P) \psi,(1-P) \gamma_{0} r\right\rangle_{\Gamma} \quad \text { for all } r \in \mathcal{R}
$$


find $U \in H^{1}(\Omega) / \mathcal{R}$ such that $P \gamma_{0} U=P \psi$ on $\Gamma$ and

$$
B(U, Y)=\int_{\Omega} \bar{Y}^{\top} f d x+\left\langle(1-P) \psi Y,(1-P) \gamma_{0}\right\rangle_{\Gamma} \quad \text { for all } Y \in{ }_{0} H^{1}(\Omega) .
$$

Here,

$$
H_{0}^{1}(\Omega) \subseteq{ }_{0} H^{1}(\Omega):=\left\{U \in H^{1}(\Omega) \mid P \gamma_{0} U=0\right\} \subseteq H^{1}(\Omega)
$$

Note that for the Dirichlet problem $P=1$ and ${ }_{0} H^{1}(\Omega)=H_{0}^{1}(\Omega)$, whereas for the Neumann problem $P=0$ and ${ }_{0} H^{1}(\Omega)=H^{1}(\Omega)$.

We assume that the differential operator $\mathcal{L}$ is strongly elliptic, i.e., it satisfies

$$
\begin{gathered}
\inf _{x \in \Omega} \sum_{j, k=1}^{d} \zeta^{\top} a_{j k}(x) \xi_{j} \xi_{k} \bar{\zeta} \geq \Lambda_{0}|\xi|^{2}|\zeta|^{2} \\
\text { for } \zeta \in \mathbb{C}^{N} \text { and } \xi=\left(\xi_{1}, \cdots, \xi_{d}\right) \in \mathbb{R}^{d} .
\end{gathered}
$$

Finally, we assume that the Garding inequality is valid on the whole space $\boldsymbol{H}^{\mathbf{1}}(\boldsymbol{\Omega})$, i.e., that there exists a positive constant $\Lambda$ so that

$$
\text { Re: } B(U, U) \geq \Lambda\|U\|_{H^{1}(\Omega)}^{2}-C(U, U) \quad \text { for all } U \in H^{1}(\Omega)
$$

where $C$ is a compact sesquilinear form on $H^{1}(\Omega) \times H^{1}(\Omega)$. For the characterization of such sytems see [30, Theorems 7.5. and 7.6]. Then the boundary value problem (2.1), (2.3) admits under assumption (2.9) a unique weak solution $U \in H^{1}(\Omega) / \mathcal{R}$ satisfying (2.10).

Let us give two examples.

Example 1: Time harmonic heat transfer in $\Omega$. Here

$$
\mathcal{L}=-\operatorname{div}(A \operatorname{grad})-\omega^{2}
$$

where the matrix $A \in \mathbb{R}^{3 \times 3}$ is symmetric and positive definite. The corresponding conormal derivative operator $(2.4)$ can be written as

$$
\gamma_{1} U=\left.n^{\top} A \nabla U\right|_{\Gamma}
$$

Example 2: Time-harmonic vibrations in a linear elastic body $\Omega$. For linear isotropic elasticity,

$$
\mathcal{L}=-\mu \triangle-(\lambda+\mu) \operatorname{grad} \operatorname{div}-\omega^{2}
$$

is the Lamé operator, where

$$
\gamma_{1} U=2 \mu \frac{\partial U}{\partial n}+\lambda(\operatorname{div} U) n+\mu n \times\left.\operatorname{curl} U\right|_{\Gamma}
$$

is the traction operator. $\lambda$ and $\mu$ are the Lamé constants. For the stationary problem, i.e., (2.3) and $\omega=0$, we have $\{0\} \subseteq \mathcal{R} \subseteq \operatorname{span}\{\vec{a}+B \wedge \vec{x}\}$, where $B=-B^{\top}$ is any real skew-symmetric matrix and $\vec{a}$ is an arbitrary constant vector. Thus, $\operatorname{dim} \mathcal{R} \leq d+d(d-1) / 2$.

Due to the Poincaré and Korn inequalities, the bilinear forms (2.7) for both examples (2.13) and (2.15) satisfy a Gårding inequality (2.12) on $H^{1}(\Omega)$, respectively.

Together with (2.1), (2.3) we will also consider the exterior boundary value problems, where (2.1) is required in $\Omega^{c}$, and $\psi$ is given on $\Gamma$. In this case, we assume in addition that the weak solution satisfies appropriate growth conditions at infinity 
which ensure uniqueness, and also that the second Green's formula holds on $\Omega^{c}$ in the form (2.8). More detailed discussion of the admissible exterior problems can be found in [5].

Remark 2.1. Assuming Green's formula (2.8) also in $\Omega^{c}$ (see [5], [6]) will be sufficient for the derivations in Section 6 [5]; but we emphasize that usually (2.8) holds in the exterior only with certain additional functionals of $U$ and $V$ supported at infinity (see [16], and for radiation conditions [38]).

We collect the regularity properties of the weak solutions $U$ for both the interior and exterior problems, and recall to this end

$$
\widetilde{\Gamma}:=\bigcup \Gamma_{j}, S:=\Gamma \backslash \widetilde{\Gamma} .
$$

Then, by standard elliptic regularity theory (see, e.g., [30] or [25]), we have

Proposition 2.2. Assume $f \in C^{\infty}(\bar{\Omega})$ and that in (2.3) we have Dirichlet data $P \psi=\varrho \in H_{\mathrm{loc}}^{s-1 / 2}\left(\Gamma_{j}\right) \cap H^{1 / 2}(\Gamma)$ and Neumann data $(I-P) \psi=\sigma \in H_{\mathrm{loc}}^{s-3 / 2}\left(\Gamma_{j}\right) \cap$ $H^{-1 / 2}(\Gamma)$ for all $j$ and with $s \geq 1$ satisfying (2.9). Then the weak solution $U$ of (2.1), (2.3) satisfies $U \in H^{s}(\Omega \backslash \overline{\mathcal{U}})$ for every open, $d$-dimensional neighborhood $\mathcal{U}$ of $S$ in $\mathbb{R}^{d}$. If, in particular, $\varrho$ and $\sigma$ are in $C^{\infty}(\widetilde{\Gamma})$, we have $U \in C^{\infty}(\bar{\Omega} \backslash \mathcal{U})$.

A stronger result is due to C.B. Morrey [29, Chap. 6.7].

Proposition 2.3. Assume that $\varrho$ and $\sigma$ in (2.3) are analytic on each surface piece $\Gamma_{j}$ and are globally in $H^{1 / 2}(\Gamma)$ or $H^{-1 / 2}(\Gamma)$, respectively, and satisfy (2.9). Then the solution $U$ of (2.1), (2.3) is analytic in $\bar{\Omega} \backslash \mathcal{U}$, where $\mathcal{U}$ is any open, non-empty neighborhood of $S$ in $\mathbb{R}^{d}$.

Remark 2.4. Under the assumptions of Proposition 2.3, $U$ admits an analytic continuation beyond the boundary surface $\Gamma_{j}$ into the exterior of $\Omega$. Consequently, also the Cauchy data $\gamma_{0} U$ and $\gamma_{1} U$ can be extended analytically beyond $\Gamma \backslash \mathcal{U}$. In the sequel, these extensions will also be denoted by $\gamma_{0} U$ and $\gamma_{1} U$.

We describe, following $[3,4,6,17]$, boundary integral equation formulations of the boundary value problems $(2.2),(2.3)$ via the so-called "direct method". The derivation of boundary integral equations of the first kind which are equivalent to the weak form of the boundary value problem and their subsequent finite element discretization goes back to $[14,31]$. We assume for simplicity that $f=0$ in (2.1). If this is not the case, a particular solution can be constructed by means of a Newtonian potential with a fundamental solution $G(x, y-x)$ of $\mathcal{L}$ defined as usual.

Definition 2.5. A (matrix) function $G(x, y-x): \mathbb{R}^{d} \times \mathbb{R}^{d} \backslash\{x=y\} \rightarrow \mathbb{C}^{N \times N}$ is a fundamental solution of $\mathcal{L}$, if

$$
\mathcal{L}\left(D_{y}\right) G(x, y-x)=\delta(y-x) I
$$

in the sense of distributions.

In the subseqent sections we need the following properties of the fundamental solution of $\mathcal{L}$.

Definition 2.6 (Pseudohomogeneous functions). A function $K(z)$ defined on $\mathbb{R}^{d} \backslash\{0\}$ is homogeneous of degree $a \in \mathbb{R}$, if $K(t z)=t^{a} K(z)$ for all $z \neq 0, t>0$. We call $K$ pseudohomogeneous of degree $a$, and write $K \in \psi h(a)$, if

$$
K(z)=K_{a}(z)+\log (|z|) Q_{a}(z)
$$


where $K_{a}$ admits an asymptotic expansion

$$
K_{a}(z) \sim \sum_{j \geq 0} K_{a j}(z)=\sum_{j \geq 0}|z|^{a+j} K_{a j}(z /|z|)
$$

with respect to homogeneous functions $K_{a j}$, and where $Q_{a}$ is a homogeneous polynomial of degree $a-d$ if $a-d \in \mathbb{N}_{0}$; and $Q_{a}=0$ otherwise. Matrix functions are pseudohomogeneous of degree $a$ if all component functions are.

Proposition 2.7. $1^{\circ}$. The fundamental solution $G(x, y-x)$ in $(2.18)$ is of the form $G(x, y-x)=K(x, x-y)$, where $K(x, z): \Omega \times \mathbb{R}^{3} \rightarrow \mathbb{C}^{N \times N}$ is a pseudohomogeneous kernel of degree -1 in $z$ for every $x \in \bar{\Omega}$ and depends analytically on $z$ on the unit sphere $|z|=1$. For every $|z|=1$, it is an analytic function of $x \in \bar{\Omega}$.

$2^{\circ}$. $Q_{a}=0$ in (2.19) for our second order systems in $\mathbb{R}^{3}$.

$3^{\circ}$ If the coefficients of $\mathcal{L}$ in (2.1) are constant, $K$ is independent of $x$.

$4^{\circ}$. If $c=0$ in $(2.2)$, then $K$ is homogeneous of degree -1 .

For a proof, see for example [19, Chapter III]. Note that for $d=2$ one has $Q_{a} \neq 0$.

Example 3: For $\mathcal{L}=-\Delta-\omega^{2}$ in $\mathbb{R}^{3}$ and $\omega \in \mathbb{C}$, we have

$$
G(x, y-x)=\frac{e^{-i \omega|x-y|}}{4 \pi|x-y|} .
$$

Example 4: For the three-dimensional Lamé operator in $(2.17)$ with $\omega=0$, the fundamental solution in $\mathbb{R}^{3}$ is the matrix function

$$
G(x, y-x)=\frac{\lambda+3 \mu}{8 \pi \mu(\lambda+2 \mu)}\left\{\frac{1}{|x-y|} I+\frac{\lambda+\mu}{\lambda+3 \mu} \frac{(x-y)(x-y)^{\top}}{|x-y|^{3}}\right\} .
$$

In the general situation, if both Cauchy data

$$
\begin{aligned}
\psi & =P \gamma_{0} U+(I-P) \gamma_{1} U, \\
\varphi & =(I-P) \gamma_{0} U+P \gamma_{1} U
\end{aligned}
$$

are known, then we have

$$
\left(\begin{array}{l}
\gamma_{0} U \\
\gamma_{1} U
\end{array}\right)=\mathcal{M}\left(\begin{array}{l}
\psi \\
\varphi
\end{array}\right):=\left(\begin{array}{cc}
P & (I-P) \\
(I-P) & P
\end{array}\right)\left(\begin{array}{l}
\psi \\
\varphi
\end{array}\right)
$$

and the solution of $(2.1),(2.3)$ is given by the representation formula

$$
U(x)=\int_{y \in \Gamma}\left\{\left({ }^{y} \gamma_{0} G\right)(x, y-x) \gamma_{1} U-\left({ }^{y} \gamma_{1} G\right)^{\top}(x, y-x) \gamma_{0} U(y)\right\} d s_{y} \text { for } x \in \Omega,
$$

where ${ }^{y} \gamma_{j}$ denotes the boundary operator $\gamma_{j}$ applied to $G$ as a function of $y$ (at fixed $x$ ). Applying ${ }^{x} \gamma_{0},{ }^{x} \gamma_{1}$ to (2.25) and utilizing the (two sided) jump relations for the single and double layer potentials (see, for example, $[4,3,6,17]$ ), we find the boundary integral relations

$$
\begin{aligned}
& \gamma_{0} U(x)=\left(\frac{1}{2} I-K\right) \gamma_{0} U(x)+\left(V \gamma_{1} U\right)(x) \text { for } x \in \widetilde{\Gamma}, \\
& \gamma_{1} U(x)=\left(D \gamma_{0} U\right)(x)+\left(\frac{1}{2} I+K^{\prime}\right) \gamma_{1} U(x) \text { for } x \in \widetilde{\Gamma} .
\end{aligned}
$$


Here, for $x$ on the boundary $\widetilde{\Gamma}$, the boundary integral operators are defined by

$$
\begin{aligned}
(V \sigma)(x) & =\int_{\Gamma}\left\{{ }^{y} \gamma_{0} G(x, y-x) \sigma(y)\right\} d s_{y}, \\
(K \varrho)(x) & =\int_{\Gamma}\left\{{ }^{y} \gamma_{1} G(x, y-x)^{\top} \varrho(y)\right\} d s_{y}, \\
\left(K^{\prime} \sigma\right)(x) & =\int_{\Gamma}\left\{{ }^{x} \gamma_{1} G(x, y-x)^{\top} \sigma(y)\right\} d s_{y}, \\
(D \varrho)(x) & =-{ }^{x} \gamma_{1} \int_{\Gamma}\left\{{ }^{y} \gamma_{1} G(x, y-x) \varrho(y)\right\} d s_{y},
\end{aligned}
$$

where, in general, the integrals are understood as an improper weakly singular integral in (2.28), as Cauchy principal value integrals in (2.29), (2.30) and as Hadamard's finite part integral in (2.31) [34].

Inserting (2.23) and (2.24), in view of the boundary conditions (2.3), we obtain two boundary integral equations for the second Cauchy datum $\varphi$, namely

$$
\begin{aligned}
P V(P \varphi) & +(I-P)\left(\frac{1}{2} I+K^{\prime}\right)(P \varphi)+(I-P) D((I-P) \varphi) \\
& +P\left(\frac{1}{2} I-K\right)((I-P) \varphi) \\
=\psi & -P\left(\frac{1}{2} I-K\right) P \psi-P V(I-P) \psi-(I-P) D P \psi \\
& -(I-P)\left(\frac{1}{2} I+K^{\prime}\right)(I-P) \psi,
\end{aligned}
$$

which is a boundary integral equation of the first kind; and

$$
\begin{aligned}
& \varphi-(I-P)\left(\frac{1}{2} I-K\right)(I-P) \varphi-(I-P) V P \varphi-P D(I-P) \varphi-\left(\frac{1}{2} I+K^{\prime}\right) P \varphi \\
& =(I-P)\left(\frac{1}{2} I-K\right) P \psi+(I-P) V(I-P) \psi-P D P \psi+P\left(\frac{1}{2} I+K^{\prime}\right)(I-P) \psi,
\end{aligned}
$$

which is a boundary integral equation of the second kind.

Remark 2.8. The integral relations (2.26), (2.27) must be modified at corner points $x \in S$; it is well known that on $S$ the factors $1 / 2$ are to be replaced by certain geometry-dependent quantities (see [2,13] and [39], for example).

Remark 2.9. In fact, for smooth $\Gamma \in C^{\infty}$, the operators $V, D, K, K^{\prime}$ are classical pseudo-differential operators of integer order.

Proposition 2.10. [4]. Let $|s| \leq 1 / 2$. Then the operators in (2.28)-(2.31) are continuous mappings in scales of Sobolev spaces on $\Gamma$. More precisely,

$$
\begin{array}{llllll}
V: & H^{-1 / 2+s}(\Gamma) & \rightarrow H^{1 / 2+s}(\Gamma), & D & : H^{1 / 2+s}(\Gamma) & \rightarrow H^{-1 / 2+s}(\Gamma), \\
K: & H^{1 / 2+s}(\Gamma) & \rightarrow H^{1 / 2+s}(\Gamma), & K^{\prime}: H^{-1 / 2+s}(\Gamma) & \rightarrow H^{-1 / 2+s}(\Gamma),
\end{array}
$$

continuously.

This proposition motivates the weak or variational form of the boundary integral equations. If we denote the parts of the non-given Cauchy data by

$$
\sigma:=P \varphi=P \gamma_{1} U, \quad \varrho:=(1-P) \varphi=(1-P) \gamma_{0} U
$$

then the weak form of the integral equations of the first kind reads: 
Find $(\sigma, \varrho) \in P H^{-1 / 2}(\Gamma) \times(1-P) H^{1 / 2}(\Gamma)$ such that

$$
\langle\widetilde{\sigma}, V \sigma\rangle_{\Gamma}-\langle\widetilde{\sigma}, K \varrho\rangle_{\Gamma}=\left\langle\widetilde{\sigma},\left\{\left(\frac{1}{2} I+K\right) P \varphi-V(1-P) \psi\right\}\right\rangle_{\Gamma}
$$

and

$$
\begin{aligned}
\left\langle\widetilde{\varrho}, K^{\prime} \sigma\right\rangle_{\Gamma}+\langle\widetilde{\varrho}, D \varrho\rangle_{\Gamma}= & \left\langle\widetilde{\varrho},\left\{\left(\frac{1}{2} I-K^{\prime}\right)(I-P) \psi-D P \psi\right\}\right\rangle_{\Gamma} \\
& \text { for all }(\widetilde{\sigma}, \widetilde{\varrho}) \in P H^{-1 / 2}(\Gamma) \times(1-P) H^{1 / 2}(\Gamma) .
\end{aligned}
$$

Remark 2.11. The integral equations are understood in the distributional sense on $\Gamma ;\langle\cdot, \cdot\rangle_{\Gamma}$ denotes the $H^{-1 / 2}(\Gamma) \times H^{1 / 2}(\Gamma)$ or $H^{1 / 2}(\Gamma) \times H^{-1 / 2}(\Gamma)$ duality pairing, respectively.

The boundary integral equations of the second kind (2.33) read:

Find $(\sigma, \varrho) \in P H^{-1 / 2}(\Gamma) \times(I-P) H^{1 / 2}(\Gamma)$ satisfying

$$
\frac{1}{2} \varrho+((I-P) K) \varrho-((I-P) V) \sigma=(I-P)(V(I-P) \psi-(I-P) K P \psi)
$$

and

$$
\frac{1}{2} \sigma-\left(P K^{\prime}\right) \sigma-P D \varrho=P\left(\left(\frac{1}{2} I+K^{\prime}\right)(I-P) \psi+D P \psi\right) .
$$

Remark 2.12. The integral operators $K$ and $K^{\prime}$ in the equations of the second kind (2.38) and (2.39) have, in general, Cauchy singular kernels and have served for a long time for solving the corresponding boundary value problems. The corresponding weak formulation, however, should use the $H^{1 / 2}(\Gamma)$-scalar product for $(2.38)$ and the $H^{-1 / 2}(\Gamma)$-scalar product for (2.39). Instead, Galerkin methods for these equations are based on the $L^{2}$-scalar product for both equations (2.38) and (2.39).

The next result shows that (2.36), (2.37) and (2.38), (2.39) are well posed and uniquely solvable.

Proposition 2.13 ([4, 3, 6, 17]). Assume that $f=0$ in (2.1). Then the integral equations (2.36), (2.37) and the boundary value problems (2.1), (2.2), respectively, are equivalent in the sense that for every given $\psi \in P H^{1 / 2}(\Gamma) \times(I-P) H^{-1 / 2}(\Gamma)$ satisfying (2.9) and a corresponding solution $U$ of the boundary value problem, $\sigma=P \gamma_{1} U, \varrho=(I-P) \gamma_{0} U$ satisfy the boundary integral equations (2.36), (2.37). Conversely, if $(\sigma, \varrho)$ is a solution of $(2.36)$, (2.37), then $U$ obtained from the representation formula (2.25) is a weak solution of (2.1).

An analogous statement holds for (2.38) and (2.39) and also for the exterior boundary value problems.

\section{Derivatives of the solution and Cauchy data on $\Gamma$}

Our purpose is the evaluation of the representation formula (2.25) and its derivatives on and near to the boundary surface $\Gamma$ by means of the Taylor formula (1.7). This will be done by utilizing the homogeneous differential equation $\mathcal{L} U=0$, valid up to the analytic boundary part $\widetilde{\Gamma}$ as in the introduction. The strong ellipticity (2.11) allows us to express the higher order normal derivatives of $U$ at $x_{0} \in \widetilde{\Gamma}$ in terms of tangential and subordinate lower normal derivatives, thereby establishing a recursive algorithm which is Cauchy's method for solving the analytic Cauchy problem. However, the tangential derivatives of the Cauchy data at $x_{0}$ are required here. 
Once more, boundary integral equations for these tangential derivatives are obtained by differentiating the boundary integral equations (2.36), (2.37) or (2.38), (2.39). Finally, we obtain a general result on the calculation of the commutators arising in this context.

3.1. Conversion of normal into tangential derivatives. In order to convert normal into tangential derivatives of the Cauchy data we observe that from (2.4) we have

$$
\gamma_{1} U=\mathcal{P}_{0} \partial_{n} U+\mathcal{Q}_{1} \gamma_{0} U
$$

where

$$
\mathcal{P}_{0}=\sum_{j, \ell=1}^{d} n_{j} a_{j \ell} n_{\ell} \quad \text { and } \quad \mathcal{Q}_{1} \gamma_{0} U=\sum_{j, \ell=1}^{d} \sum_{\nu, \iota=1}^{d-1} n_{j} a_{j \ell} \frac{\partial \chi_{\ell}}{\partial v_{\nu}} \gamma^{\nu \iota} \frac{\partial \gamma_{0} U}{\partial v_{\iota}}
$$

Here, $\mathcal{P}_{0}$ is a matrix function which is $C^{\infty}$ or even analytic on $\widetilde{\Gamma}$ and invertible in the tubular neighborhood $\mathcal{U}\left(\Gamma_{j}\right)$, due to the strong ellipticity condition (2.11); and $\mathcal{Q}_{1}$ is there a tangential operator of order 1 with smooth, respectively analytic coefficients.

Theorem 3.1. Let $k \geq 0$ be an integer, and let $U$ satisfy $\mathcal{L} U=0$ in $\Omega \cup \widetilde{\Gamma}$ with Cauchy data $\gamma_{0} U, \gamma_{1} U$ analytic or sufficiently differentiable on $\widetilde{\Gamma}$. Then any tangential derivative $\partial^{\alpha}$ of arbitrary order $|\alpha|$ of $\partial_{n}^{k} U$ at $x_{0} \in \widetilde{\Gamma}$ can be expressed exclusively by tangential derivatives of the Cauchy data $\gamma_{0} U$ and $\gamma_{1} U$ in $x_{0}$.

Proof. The proof is by induction and corresponds to Cauchy's algorithm for solving the Cauchy problem.

For $k=0$, the assertion is obvious.

For $k=1$, we differentiate

$$
\partial_{n} U=\mathcal{P}_{0}^{-1}\left(\gamma_{1} U-\mathcal{Q}_{1} \gamma_{0} U\right)
$$

tangentially. Then the Leibniz formula and smoothness of $\mathcal{P}_{0}^{-1}$ yield the assertion.

For $k=2$ we use the differential equation in the tubular neighbourhood of $\mathcal{U}\left(\Gamma_{j}\right)$ introduced in Proposition 1.1; i.e.,

$$
0=\mathcal{L} U=-\sum_{j, k=1}^{d} D_{j} a_{j k}(x) D_{k} U+c U=-\mathcal{P}_{0} \partial_{n}^{2} U-\mathcal{P}_{1} \partial_{n} U-\mathcal{P}_{2} U
$$

Here, $\mathcal{P}_{i}$ are tangential differential operators of order $i$ with coefficients which are analytic in $\mathcal{U}\left(\Gamma_{j}\right)$ given by $\mathcal{L}$ and the geometry of $\Gamma_{j}$. (The explicit formulae are presented in the Appendix.) From (3.1) and (3.2) we find on $\widetilde{\Gamma}$

$$
\partial_{n}^{2} U=-\mathcal{P}_{0}^{-1}\left\{\mathcal{P}_{1} \mathcal{P}_{0}^{-1}\left(\gamma_{1} U-\mathcal{Q}_{1} \gamma_{0} U\right)+\mathcal{P}_{2} \gamma_{0} U\right\}
$$

Differentiating (3.3) tangentially and using the Leibniz rule proves the assertion.

For the induction step, fix $K>2$ and assume that the theorem holds for $0 \leq$ $k \leq K-1$. Apply $\partial_{n}^{K-2}$ to (3.2), i.e.,

$$
\begin{aligned}
\partial_{n}^{K} U & =-\partial_{n}^{K-2} \mathcal{P}_{0}^{-1}\left\{\mathcal{P}_{1} \partial_{n} U+\mathcal{P}_{2} U\right\} \\
& =-\sum_{\ell=0}^{K-2}\left(\begin{array}{c}
K-2 \\
\ell
\end{array}\right)\left(\partial_{n}^{\ell} \mathcal{P}_{0}^{-1}\right) \partial_{n}^{K-2-\ell}\left\{\mathcal{P}_{1} \partial_{n} U+\mathcal{P}_{2} U\right\}
\end{aligned}
$$


Since

$$
\partial_{n}^{K-2-\ell} \mathcal{P}_{i}=\sum_{t=0}^{K-2-\ell} \mathcal{P}_{i, t} \partial_{n}^{t}
$$

where $\mathcal{P}_{i, t}$ are tangential differential operators of orders $\leq i=1,2$, we obtain

$$
\partial_{n}^{K} U=-\sum_{\ell=0}^{K-2}\left(\begin{array}{c}
K-2 \\
\ell
\end{array}\right)\left(\partial_{n}^{\ell} \mathcal{P}_{0}^{-1}\right) \sum_{t=0}^{K-2-\ell}\left\{\mathcal{P}_{1, t} \partial_{n}^{t+1} U+\mathcal{P}_{2, t} \partial_{n}^{t} U\right\},
$$

where the highest order of normal derivatives on the right-hand side is $K-1$. Further tangential differentiation of (3.5) and the Leibniz rule together with the induction assumption yield the proposed representation of $\partial^{\alpha} \partial_{n}^{K} U$ by a linear combination of $\partial^{\beta} \gamma_{1} U$ and $\partial^{\mu} \gamma_{0} U$ for $|\beta| \leq|\alpha|+K-1$ and $|\mu| \leq|\alpha|+K$.

\subsection{Boundary integral equations for tangential derivatives of the Cauchy} data.

Lemma 3.2. Let $A$ be a classical pseudo-differential operator of integer order a on $\Gamma \subset \mathbb{R}^{3}$. Then

$$
\partial_{1}^{k} \partial_{2}^{\ell} A \varphi=\sum_{i=0}^{k} \sum_{j=0}^{\ell}\left(\begin{array}{c}
k \\
i
\end{array}\right)\left(\begin{array}{l}
\ell \\
j
\end{array}\right) A_{(i, j)}\left(\partial_{1}^{k-i} \partial_{2}^{\ell-j} \varphi\right),
$$

where the commutators $A_{(i, j)}$ are defined recursively by

$$
\begin{aligned}
& A_{(0,0)}:=A, \\
& A_{(i+1, j)}:=\partial_{1} A_{(i, j)}-A_{(i, j)} \partial_{1}, \\
& A_{(i, j+1)}=\partial_{2} A_{(i, j)}-A_{(i, j)} \partial_{2} .
\end{aligned}
$$

Every $A_{(i, j)}$ is a classical pseudo-differential operator of order a on $\Gamma$.

Clearly, for $d=2$, i.e., $\Gamma \subset \mathbb{R}^{2}$, these formulae simplify accordingly.

Proof. The representation (3.6) follows from the definition (3.7) of the commutators and the binomial formula by elementary induction. Also recursively, from (3.7) follows the assertion on the order $a$ with [37, Corollary 4.2], since the differential operators are scalar operators.

This lemma will now be applied to the boundary integral equations $(2.36),(2.37)$, respectively $(2.38),(2.39)$, which we write in the generic form

$$
A \varphi=F \psi
$$

for the missing Cauchy datum $\varphi$. We now assume that the integral operator $A$ in (3.8) is injective. Generically (but not always), this is the case when the original boundary value problem $(2.1),(2.3)$ is uniquely solvable. For the equations of the first kind (2.36) and (2.37), the Gårding inequality (2.12) in the domain together with its variant for the exterior domain implies the Gårding inequality

$$
\operatorname{Re}\langle\varphi, A \varphi\rangle \geq \Lambda_{1}\|\varphi\|_{0}^{2}-\operatorname{Re} C(\varphi, \varphi) \text { for all } \varphi \in \mathcal{H}^{0}
$$

(see [6, Theorem 3.9]), where

$$
\mathcal{H}^{t}:=P H^{-1 / 2+t}(\Gamma) \times(I-P) H^{1 / 2+t}(\Gamma)
$$

equipped with the norm

$$
\|\varphi\|_{t}=\|(\sigma, \varrho)\|_{t}:=\|\sigma\|_{H^{-1 / 2+t}(\Gamma)}+\|\varrho\|_{H^{1 / 2+t}(\Gamma)}
$$




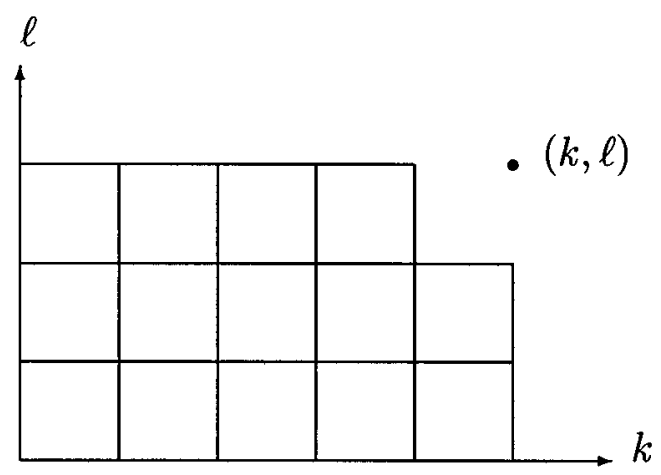

FiguRE 2. Dependency graph

and where $C(\cdot, \cdot): \mathcal{H}^{0} \times \mathcal{H}^{0} \rightarrow \mathbb{C}$ is a compact sesquilinear form. For $\varphi=(\sigma, \varrho)$ and $\widetilde{\varphi}=(\widetilde{\sigma}, \widetilde{\varrho}) \in \mathcal{H}^{0}$, the pairing $\langle\widetilde{\varphi}, \varphi\rangle$ is defined by

$$
\langle\widetilde{\varphi}, \varphi\rangle:=\langle\widetilde{\sigma}, \varrho\rangle_{\Gamma}+\langle\widetilde{\varrho}, \sigma\rangle_{\Gamma} .
$$

For the equations of the second kind $(2.38),(2.39)$ we consider $\langle\cdot, \cdot\rangle$ to be the $L_{2}$-scalar product and take $\mathcal{H}^{t}:=H^{t}(\Gamma)$. In this case we assume (3.9) to hold, which must be verified for the particular problem at hand. (For details we refer to [40].)

In practice, either type of integral equations can be used for solving the boundary value problem and as the basic formulation for its numerical solution.

To obtain a boundary integral equation for tangential derivatives of $\varphi$, we differentiate both sides of (3.8) and regroup terms. The boundary integral equation for $\partial^{\alpha} \varphi:=\partial_{1}^{k} \partial_{2}^{\ell} \varphi$ with $\alpha=(k, \ell)$ reads

$$
A \partial^{\alpha} \varphi=F \partial^{\alpha} \psi+\sum_{0<\beta \leq \alpha}\left(\begin{array}{l}
\alpha \\
\beta
\end{array}\right)\left\{F_{(\beta)} \partial^{\alpha-\beta} \psi-A_{(\beta)} \partial^{\alpha-\beta} \varphi\right\},
$$

where $\alpha=(k, \ell), \beta=(i, j),\left(\begin{array}{c}\alpha \\ \beta\end{array}\right)=\left(\begin{array}{l}k \\ i\end{array}\right)\left(\begin{array}{l}\ell \\ j\end{array}\right)$ and $0<\beta \leq \alpha$ means $(1 \leq i+j) \wedge(0 \leq$ $i \leq k) \wedge(0 \leq j \leq \ell)$.

Note particularly, that the integral operator $A$ in (3.13) is the same as in (3.8). Therefore, any available discretization of (3.8) can readily be used to solve (3.13). Recursive use of (3.13) allows the computation of $\partial^{\alpha} \varphi$ for any $\alpha=(k, \ell) \in \mathbb{N}_{0}^{2}$. To see this, consider the dependency graph in Figure 2.

Each derivative corresponds to a grid point in the $k-\ell-$ plane. For fixed $(k, \ell)$ corresponding to $\bullet$ in the figure, all derivatives in the shaded region are generally needed. Starting with $(0,0)$, i.e., equation (3.8), compute $\varphi$; then a boot-strapping procedure with rectangles of increasing size allows us to reach any $\alpha=(k, \ell)$.

In the two-dimensional case $d=2$, all the indices $\alpha, \beta$ in (3.13) are only scalar indices.

Remark 3.3. The integral operators in (3.8) and (3.13) must, for computational purposes, generally be written in the local coordinates of the charts $\chi_{j}$ which are assumed to form a piecewise $C^{\infty}$-atlas or sufficiently differentiable atlas of $\Gamma$; see also Remark 3.7, below. 
3.3. Calculation of the commutators. As mentioned in Lemma 3.2, all the commutators in (3.13) are classical pseudo-differential operators whose orders are equal to those of $A$ and $F$, respectively.

Since $A$ and $F$ were obtained by boundary reduction, these operators admit in each chart the representation with "finite part", Cauchy singular and weakly singular integrals in connection with non-integrable kernels in the boundary integral operators (see [34]).

Definition 3.4. Let $\varepsilon \rightarrow A(\varepsilon)$ denote a complex-valued function which is continuous on $\left(0, \varepsilon_{0}\right)$ for some $\varepsilon_{0}>0$ and admits an asymptotic expansion of the form

$$
A(\varepsilon)=A_{0}+A_{1} \log \varepsilon+\sum_{j=2}^{m} A_{j} \varepsilon^{-j+1}+o(1)
$$

as $\varepsilon \rightarrow 0$, where $A_{j} \in \mathbb{C}$. Then $A_{0}$ is called the finite part of $A(\varepsilon)$, and we write $A_{0}=$ p.f. $A(\varepsilon)$.

The finite part in (3.14) is well-defined, since

$$
C_{0}+C_{1} \log \varepsilon+\sum_{j=2}^{m} C_{j} \varepsilon^{-j+1}=o(1)
$$

as $\varepsilon \rightarrow 0$ implies that $C_{0}=C_{1}=\ldots=C_{m}=0$. For a detailed treatment of the finite part concept in conjunction with two-dimensional area and surface integrals with non-integrable kernels, we refer to [34]. There one can also find the behaviour of finite part integrals under the substitution of variables.

Since $A$ and $F$ are matrices of classical pseudo-differential operators, their entries $B_{n}$ in local coordinates on $\Gamma_{j}$ have the form

$$
B_{n} \varphi=\wp_{n} \varphi+\text { p.f. } \int_{V_{j}} \widetilde{K}_{n}(u, v-u) \varphi(v) d v \quad \text { for } \varphi \in C_{0}^{\infty}\left(\Gamma_{j}\right),
$$

where

$$
\wp_{n} \varphi=\sum_{|\alpha| \leq n} b_{\alpha}(u) \partial^{\alpha} \varphi(u) .
$$

The subscript $n$ denotes the order of $B_{n}$, and $\wp_{n} \equiv 0$ if $n<0$. Our aim is a representation of the commutator of $B_{n}$ with tangential differentiation. Since, for $\wp_{n}$, the commutators

$$
\partial^{\alpha} \wp_{n} \varphi-\wp_{n} \partial^{\alpha} \varphi \quad \text { for }|\alpha|=1
$$

can be computed in the usual fashion, we consider only the integral operator in (3.15). Our main result is as follows.

Theorem 3.5. Let $\varphi \in C_{0}^{\infty}\left(\Gamma_{j}\right)$, and let $\partial^{\lambda}$ be any tangential differential operator of order $|\lambda|=1$. Then, in the local coordinates induced by the chart $\chi_{j}$, we have

$$
\left[\partial^{\lambda}, B_{n}\right] \varphi(u)=\left[\partial^{\lambda}, \wp_{n}\right] \varphi(u)+\text { p.f. }\left.\int_{V_{j}}\left(\partial_{u}^{\lambda} \widetilde{K}_{n}(u, t)\right)\right|_{t=v-u} \varphi(v) d v
$$


Proof. The kernel function $\widetilde{K}_{n}(u, t)$ with $t=v-u$ is $C^{\infty}$ with respect to $u$ for all $t \neq 0$ and is pseudohomogeneous in $t$ of order $-n-2$. In particular, for any $L \geq 0$,

$$
\widetilde{K}_{n}(u, t)=\sum_{0 \leq j \leq L} \widetilde{k}_{n-j}(u, t)+\widetilde{K}_{n-L-1}(u, t)
$$

with

$$
\widetilde{k}_{n-j}(u, t)=r^{-2-n+j} \widetilde{f}_{n-j}(u, \Theta)
$$

and

$$
\widetilde{K}_{n-L-1}(u, t)=\mathcal{O}\left(r^{L-n-1}\right) \quad \text { uniformly in } u,
$$

where $t=r(\cos \Theta, \sin \Theta)^{\top}$. Here, for $d=3$, logarithmic terms are absent since here we consider only boundary integral equations for second order elliptic boundary value problems in $\mathbb{R}^{3}$. In what follows we choose $L>n$.

Due to (3.18) we will show (3.17) separately for the remainder $\widetilde{K}_{n-L-1}(u, t)$ and the homogeneous terms $\widetilde{k}_{n-j}$.

For $\widetilde{K}_{n-L-1}(u, t)$ we use that $\partial_{u}^{\lambda} \widetilde{K}_{K-L-1}(u, v-u)$ is only weakly singular and, hence, integrable. Therefore,

$$
\partial_{u}^{\lambda} \int_{V_{j}} \widetilde{K}_{n-L-1}(u, v-u) \varphi(v) d v=\int_{V_{j}}\left(\partial_{u}^{\lambda} \widetilde{K}_{n-L-1}(u, v-u)\right) \varphi(v) d v .
$$

Now we use the elementary property

$$
\partial_{u}^{\lambda}\left(\widetilde{K}_{n-L-1}(u, v-u)\right)=\left(\partial_{u}^{\lambda} \widetilde{K}_{n-L-1}(u, t)\right)_{\left.\right|_{t=v-u}}-\partial_{v}^{\lambda} \widetilde{K}_{n-L-1}(u, v-u),
$$

insert it into (3.18) and integrate the last term in the resulting integral by parts. Since $\varphi \in C_{0}^{\infty}\left(V_{j}\right)$, the corresponding line integral vanishes, and (3.18) with (3.20) yields the assertion (3.17) for $\widetilde{K}_{K-L-1}$.

For the remaining terms choose $R>0$ such that the ball $B_{R}(u):=\{v|| v-u \mid \leq$ $R\}$ satisfies $B_{R}(u) \subset V$; and choose an associated cut-off function $\chi \in C_{0}^{\infty}\left(B_{r}(u)\right)$ with $\chi(v) \equiv 1$ for $|v-u| \leq R / 2$. Note, however, that $\chi$ does not depend on $u$. Set

$$
w:=\chi \varphi \in C_{0}^{\infty}\left(B_{R}(u)\right) ; \varphi=w+(1-\chi) \varphi .
$$

Now consider the term

$$
\widetilde{k}_{n-j}(u, t)=r^{-2-n+j} f_{n-j}(u, \Theta),
$$

where $\Theta=\frac{v-u}{r}, r=|v-u|$. The corresponding operator is given by

$$
\begin{aligned}
B_{n-j} \varphi & =B_{n-j} w+B_{n-j}(1-\chi) \varphi \\
B_{n-j} w(u)= & \int_{r \leq R} \frac{f_{n-j}(u, \Theta)}{r^{n+2-j}}\left\{w(v)-\sum_{0 \leq|\beta| \leq n-j} \frac{(v-u)^{\beta}}{\beta !}\left(\partial_{u}^{\beta} w(u)\right)\right\} d v \\
& +\sum_{0 \leq|\beta| \leq n-j} c_{\beta}(u) \partial^{\beta} w(u),
\end{aligned}
$$

and

$$
B_{n-j}(1-\chi) \varphi(u)=\int_{\operatorname{supp}((1-\chi) \varphi)} \widetilde{k}_{n-j}(u, v-u)((1-\chi) \varphi(v)) d v
$$


Since the integrand in (3.23) is $C_{0}^{\infty}$, we find, analogously to (3.20), that

$$
\begin{aligned}
\partial_{u}^{\lambda} B_{n-j}(1-\chi) \varphi(x)= & \left.\int_{\operatorname{supp}((1-\chi) \varphi)}\left(\partial_{u}^{\lambda} \widetilde{k}_{n-j}(u, t)\right)\right|_{t=v-u}((1-\chi) \varphi(v)) d v \\
& -\int_{\operatorname{supp}((1-\chi) \varphi)}\left(\partial_{v}^{\lambda} \widetilde{k}_{n-j}(u, v-u)\right)((1-\chi) \varphi(v)) d v
\end{aligned}
$$

Integrating the last term again by parts, one gets

$$
\left[\partial^{\lambda}, B_{n-j}((1-\chi) \varphi)\right]=\left.\int_{V}\left(\partial_{u}^{\lambda} \widetilde{k}_{n-j}(u, t)\right)\right|_{t=v-u}((1-\chi) \varphi) d v
$$

It remains to analyze (3.22). First, note that

$$
\begin{aligned}
c_{\beta}(u) & =\text { p.f. } \int_{B_{R}} \frac{f_{n-j}(u ; \Theta)}{r^{n+2-j}} \frac{(v-u)^{\beta}}{\beta !} d v \\
& =\int_{\vartheta=0}^{2 \pi} \frac{f_{n-j}(u ;(\cos \vartheta, \sin \vartheta))}{\beta !} \pi_{\beta}(\vartheta) d \vartheta \times \begin{cases}\ln R & \text { if } j+|\beta|=n, \\
\frac{R^{j+|\beta|-n}}{j+|\beta|-n} & \text { else, }\end{cases}
\end{aligned}
$$

and

$$
\pi_{\beta}(\vartheta)=(\cos \vartheta, \sin \vartheta)^{\beta}:=\cos ^{\beta_{1}} \vartheta \sin ^{\beta_{2}} \vartheta \quad \text { with } \beta \in \mathbb{N}_{0}^{2} .
$$

Differentiating (3.22), we get for $|\lambda|=1$

$$
\begin{aligned}
\partial^{\lambda} B_{n-j} w(u)= & \partial^{\lambda} \int_{r \leq R} \frac{1}{r} H(u, v) d v \\
& +\sum_{0 \leq|\beta| \leq n-j}\left\{\left(\partial^{\lambda} c_{\beta}(u)\right) \partial^{\beta} w(u)+c_{\beta}(u) \partial^{\lambda+\beta} w(u)\right\}
\end{aligned}
$$

with

$$
H(u, v)=f_{n-j}(u, \Theta) r^{-n+j-1}\left\{w(v)-\sum_{0 \leq|\beta| \leq n-j} \frac{(v-u)^{\beta}}{\beta !} \partial^{\beta} w(u)\right\} .
$$

Since $H$ is bounded, the integral in (3.27) is weakly singular. Using the rule of differentiation for weakly singular integrals (see $[27$, Section 8$]$ ), we find that, for every $0<\varepsilon<R$,

$$
\partial^{\lambda} \int_{\varepsilon \leq r \leq R} \frac{1}{r} H(u, v) d v=\int_{\varepsilon \leq r \leq R} \partial_{u}^{\lambda}\left(\frac{1}{r} H(u, v)\right) d v-\int_{|v-u|=\varepsilon} H(u, v)(v-u)^{\lambda} \varepsilon^{-2} d s .
$$


We calculate

$$
\begin{aligned}
\partial_{u}^{\lambda}\left(\frac{1}{r} H(u, v)\right)= & \left.\left(\partial_{u}^{\lambda} f_{n-j}(u, \Phi)\right)\right|_{\Phi=\Theta} r^{-n+j-2}\{\cdots\} \\
& +\left.\left(\partial_{u}^{\lambda} \frac{f_{n-j}(s, \Theta)}{r^{n-j+2}}\right)\right|_{s=u}\{\cdots\} \\
& +\frac{f_{n-j}(u, \Theta)}{r^{n-j+2}} \sum_{1 \leq|\beta| \leq n-j}(\beta \cdot \lambda) \frac{(v-u)^{\beta-\lambda}}{\beta !} \partial^{\beta} w(u) \\
& -\frac{f_{n-j}(u, \Theta)}{r^{n-j+2}} \sum_{1 \leq|\beta| \leq n-j} \frac{(v-u)^{\beta}}{\beta !} \partial^{\beta+\lambda} w(u) .
\end{aligned}
$$

Since $\Theta=\frac{1}{r}(v-u)$ and $r=|v-u|$, one has

$$
\partial_{u}^{\lambda} \frac{f_{n-j}(s, \Theta)}{r^{n-j+2}}=-\partial_{v}^{\lambda} \frac{f_{n-j}(s, \Theta)}{r^{n-j+2}} .
$$

We use (3.30) in the second term of (3.29) and integrate the corresponding integral by parts to obtain

$$
\begin{aligned}
& \left.\int_{\varepsilon<r \leq R}\left(\partial_{u}^{\lambda} \frac{f_{n-j}(s, \Theta)}{r^{n-j+2}}\right)\right|_{s=u}\{\cdots\} d v \\
& \quad=\int_{\varepsilon<r \leq R} \frac{f_{n-j}(s, \Theta)}{r^{n-j+2}} \partial_{v}^{\lambda}\{\cdots\} d v+\int_{|v-u|=\varepsilon} H(u, v)(v-u)^{\lambda} \varepsilon^{-2} d s
\end{aligned}
$$

since supp $w \subset B_{R}(u)$. Inserting (3.29) into (3.28) and using (3.31), we get

$$
\begin{aligned}
& \partial^{\lambda} \int_{\varepsilon<r \leq R} \frac{1}{r} H(u, v) d v=\left.\int_{\varepsilon<r \leq R}\left(\partial_{u}^{\lambda} f_{n-j}(u, \Phi)\right)\right|_{\Phi=\Theta} r^{-n+j-2}\{\cdots\} d v \\
& \quad+\int_{\varepsilon<r \leq R} \frac{f_{n-j}(s, \Theta)}{r^{n-j+2}}\left\{\partial_{v}^{\lambda} w(v)-\sum_{|\beta| \leq n-j} \frac{(v-u)^{\beta}}{\beta !} \partial_{n}^{\beta+\lambda} w(u)\right\} d v .
\end{aligned}
$$

Since the integrals in (3.32) are weakly singular, they depend on $\varepsilon$ continuously and (3.32) holds for $\varepsilon=0$, too. Inserting (3.32) in (3.27), we find that

$$
\begin{aligned}
& \partial^{\lambda} B_{n-j} w(u)=\left(B_{n-j} \partial^{\lambda} w(u)\right)+\sum_{|\beta| \leq n-j}\left(\partial^{\lambda} c_{\beta}(u)\right) \partial^{\beta} w(u) \\
& +\left.\int_{\varepsilon<r \leq R}\left(\partial_{u}^{\lambda} f_{n-j}(u, \Phi)\right)\right|_{\Phi=\Theta} r^{-n+j-2}\left\{w(v)-\sum_{|\beta| \leq n-j} \frac{(v-u)^{\beta}}{\beta !} \partial_{u}^{\beta+\lambda} w(u)\right\} d v .
\end{aligned}
$$


Note that from (3.27) we have

$$
\begin{aligned}
\partial^{\lambda} c_{\beta}(u) & =\left.\int_{0}^{2 \pi}\left(\partial_{u}^{\lambda} f_{n-j}(u, \Phi)\right)\right|_{\Phi=\Theta(\vartheta)} \frac{\pi_{\beta}(\vartheta)}{\beta !} d \vartheta \times\left\{\begin{array}{l}
\ln R \text { if } j+|\beta|=n, \\
\frac{R^{j+|\beta|-n}}{j+|\beta|-n} \text { else },
\end{array}\right. \\
& =\text { p.f. }\left.\int_{0<r \leq R}\left(\partial_{u}^{\lambda} f_{n-j}(u, \Phi)\right)\right|_{\Phi=\Theta} r^{-n+j-2} \frac{(v-u)^{\beta}}{\beta !} d v,
\end{aligned}
$$

which gives in (3.29) the desired equation for the special operator in (3.22) with (3.26). Hence,

$$
D^{\lambda} B_{n-j} w(u)=B_{n-j} D^{\lambda} w(u)+\text { p.f. } \int_{V_{j}}\left(D_{u}^{\lambda} f_{n-j}(u, \varphi)\right)_{\mid \varphi=\Theta} r^{-n+j-2} w(v) d v .
$$

Summing (3.34) and (3.23) over $0 \leq j \leq L$, adding it to (3.20) and referring to (3.18) completes the proof.

Corollary 3.6. Let $T \subset \subset V_{j}$ be some compact, simply connected and piecewise smoothly bounded subregion of $V_{j}$ with $u \in \stackrel{\circ}{T}$, and let $\varphi \in C^{\infty}\left(\chi_{j}(T)\right)$. Let $\partial^{\lambda}$ be any differential operator of order $|\lambda|=1, \lambda=\left(\lambda_{1}, \lambda_{2}\right)$ with $\lambda_{k} \in\{0,1\}, k=1,2$. Then for the operator

$$
B_{n T} \varphi=\wp_{n} \varphi+\text { p.f. } \int_{T} \widetilde{K}_{n}(u, v-u) \varphi(v) d v
$$

we have the commutator property

$$
\begin{aligned}
{\left[\partial^{\lambda}, B_{n T}\right] \varphi(u)=\left[\partial^{\lambda}, \wp_{n}\right] \varphi(u) } & + \text { p.f. }\left.\int_{T}\left(\partial_{u}^{\lambda} \widetilde{K}_{n}(u, t)\right)\right|_{t=v-u} \varphi(v) d v \\
& -\oint_{v \in \partial T} \widetilde{K}_{n}(u, v-u) \varphi(v)(\widetilde{n}(v) \cdot \lambda) d \gamma
\end{aligned}
$$

Here $\gamma$ denotes the parameter of arc-length on $\partial T$, and $\widetilde{n}(v)$ the exterior twodimensional normal vector of $\partial T$.

Proof. For the proof we write $\varphi(u)=w(u)+\varphi_{1}(u)$ as in (3.21) and get (with $\varphi_{1}=(1-\chi) \varphi$ as in $\left.(3.24)\right)$

$$
\begin{aligned}
\partial_{n}^{\lambda} B_{n T} \varphi_{1}= & \left.\int_{\operatorname{supp}\left(\varphi_{1}\right) \cap T}\left(\partial_{u}^{\lambda} \widetilde{K}_{n}(u, t)\right)\right|_{t=v-u} \varphi_{1}(v) d v \\
& -\int_{\operatorname{supp}\left(\varphi_{1}\right) \cap T}\left(\partial_{v}^{\lambda} \widetilde{K}_{n}(u, v-u)\right) \varphi_{1}(v) d v .
\end{aligned}
$$

Integration by parts of the last term yields (3.36) for $\varphi=\varphi_{1}$, since $\varphi_{1}$ is identically zero in $B_{R / 2}(u)$. For $w \in C_{0}^{\infty}(T)$ we apply Theorem 3.5, which completes the proof. 
Remark 3.7. So far we have considered only the three-dimensional case. All assertions, however, have exact analogues in two dimensions where $\Gamma$ is a piecewise $C^{\infty}$-curve.

The details for the two-dimensional case can be found in [32].

Example 5: As an example for the application of the commutator formula (3.17) consider the single layer potential

$$
V \varphi= \begin{cases}-\frac{1}{2 \pi} \int_{\Gamma} \ln |x-y| \varphi(y) d s_{y} & \text { in } \mathbb{R}^{2}, \\ \frac{1}{4 \pi} \int_{\Gamma}|x-y|^{-1} \varphi(y) d s_{y} & \text { in } \mathbb{R}^{3} .\end{cases}
$$

Here we have, of course, $\wp_{n} \equiv 0$ since $n=-1$.

The commutators are as follows:

In 2-D:

$$
V_{(1)} \varphi=\left[\frac{d}{d s}, V\right] \varphi=\frac{1}{2 \pi} \int_{\Gamma} \frac{(y-x) \cdot\left(\chi^{\prime}(y)-\chi^{\prime}(x)\right)}{|y-x|^{2}} \varphi(y) d s_{y},
$$

where $\chi^{\prime}$ denotes the tangential vector defined by the derivative to $s$, the parameter of the arc length on $\Gamma$.

In $3-D$ for $|\lambda|=1$ :

$$
\begin{aligned}
V_{(\lambda)} \varphi & =\left[\partial^{\lambda}, V\right] \varphi \\
& =\frac{1}{4 \pi} \int_{\Gamma}\left\{\frac{(y-x) \cdot\left(\chi_{\mid \lambda}(y)-\chi_{\mid \lambda}(x)\right)}{|y-x|^{3}}+\frac{1}{|x-y|}(\log \sqrt{\gamma})_{\mid \lambda}\right\} \varphi(y) d s_{y} .
\end{aligned}
$$

\section{Numerical APPRoXimation OF the DERIVATIVES}

In the present section we analyze Galerkin discretizations for the system of boundary integral equations (3.13). The basic idea is that any stable discretization of the original equation (3.8) is also applicable to the system (3.13), since the latter is triangular. This yields numerical approximations and corresponding error estimates for the differentiated Cauchy data $\partial^{\alpha} \varphi$. We consider only Galerkin schemes based on $h$-refinements on the boundary $\Gamma$. It should be clear, however, that all the arguments carry over to more general Galerkin-Petrov projection schemes (as e.g., least squares methods, Nyström methods and collocation methods) as well as to more general approximation schemes such as $p$ - and $h p$-versions of the BEM.

The error estimates are obtained in the usual scales of Sobolev norms. To exploit the Taylor formula (1.7), however, pointwise approximations of $\partial^{\alpha} \varphi$ are needed. To this end, we use an extraction formula based on the Riesz potentials and present corresponding pointwise error estimates.

4.1. Galerkin schemes for tangential derivatives. As usual, a Galerkin approximation of (3.8) reads: Find $\varphi_{h} \in \mathcal{H}_{h}$ such that

$$
\left\langle\widetilde{\varphi}_{h}, A \varphi_{h}\right\rangle_{\Gamma}=\left\langle\widetilde{\varphi}_{h}, F \psi\right\rangle_{\Gamma} \quad \text { for all } \widetilde{\varphi}_{h} \in \mathcal{H}_{h} .
$$

Here $\mathcal{H}_{h}$ is a family of finite-dimensional subspaces of $P H^{-1 / 2}(\Gamma) \times(1-P) H^{1 / 2}(\Gamma)$ providing the approximation property

$$
\inf _{\widetilde{\varphi}_{h} \in \mathcal{H}_{h}}\left\|\varphi-\widetilde{\varphi}_{h}\right\|_{\mathcal{H}^{t}} \leq c h^{\tau-t}\|\varphi\|_{\mathcal{H}^{\tau}} \quad \text { for } t \leq \tau .
$$


For example, for the integral equations of the first kind (2.36) and (2.37) we select

$$
\mathcal{H}_{h}=P S_{h}^{d, r} \times(1-P) S_{h}^{d+1, r+1},
$$

where $S_{h}^{d, r}$ with $t \leq r<d$ denotes a family of finite elements on the boundary in the sence of Babuška and Aziz [1]; then $\tau \leq d+\frac{1}{2}$ in (4.2).

For the integral equations of the second kind (2.38) and (2.39) we may select $\mathcal{H}_{h}:=S_{h}^{d, r} \subset \mathcal{H}^{0}=L_{2}(\Gamma)$; then $\tau \leq d$ in (4.2).

As is well known, the Gårding inequality (3.9) and injectivity of $A$ provide quasioptimal asymptotic convergence, i.e.,

$$
\left\|\varphi-\varphi_{h}\right\|_{t} \leq c h^{\tau-t}\|\varphi\|_{\tau}
$$

for $-d-\frac{1}{2} \leq t \leq 0 \leq \tau \leq d+\frac{1}{2}$ in case of equations of the first kind (2.36), (2.37) and for $-d \leq t \leq 0 \leq \tau \leq d$ in case of equations of the second kind (2.38), (2.39) (see $[15])$.

To approximate the derivative $\partial^{\alpha} \varphi$ of the Cauchy data $\varphi$ for a given $\alpha=(k, \ell)$, we introduce the vector $\vec{\varphi}=\left\{\partial^{\delta} \varphi\right\}$ of partial derivatives of orders $\delta=(i, j), 0 \leq$ $i \leq k \wedge 0 \leq j \leq \ell$. We order the components of $\vec{\varphi}$ according to ascending orders $i+j$. This is a partial ordering, denoted by $\delta_{m}$ with $m=1, \ldots, M=(k+1)(\ell+1)$. Then (3.13) can be written as

$$
A \partial^{\delta_{m}} \varphi+\sum_{0<\beta \leq \delta_{m}}\left(\begin{array}{c}
\delta_{m} \\
\beta
\end{array}\right) A_{(\beta)} \partial^{\delta_{m}-\beta} \varphi=\partial^{\delta_{m}}(F \psi), \quad m=1, \ldots, M,
$$

or as a triangular system of equations

$$
\mathcal{A} \vec{\varphi}=\vec{f}:=\left(\partial^{\delta_{m}} F \psi\right)_{m=1}^{M}
$$

with the diagonal $\mathcal{A}_{m m}=A$.

Lemma 4.1. There exists a positive diagonal constant matrix $\Theta \in \mathbb{R}^{M} \times \mathbb{R}^{M}$ such that

$$
\operatorname{Re}\langle\Theta \mathcal{A} \vec{\varphi}, \vec{\varphi}\rangle \geq \Lambda_{0}\|\vec{\varphi}\|_{\left(\mathcal{H}^{0}\right)^{M}}^{2}-\operatorname{Re} \mathcal{C}(\vec{\varphi}, \vec{\varphi}) \quad \text { for all } \vec{\varphi} \in\left(\mathcal{H}^{0}\right)^{M}
$$

where $\Lambda_{0}>0$ and $\mathcal{C}:\left(\mathcal{H}^{0}\right)^{M} \times\left(\mathcal{H}^{0}\right)^{M} \rightarrow \mathbb{C}$ is a compact sesquilinear form.

The duality pairing in (4.7) is the natural extension of $\langle\cdot, \cdot\rangle$ to the vector case.

Proof. The proof is by induction with respect to $M$. The case $M=1$ corresponds to one equation and (3.9). Now assume that (4.7) holds for some $M_{0} \geq 1$, i.e.,

$\operatorname{Re}\left\langle\Theta_{M_{0}} \mathcal{A}_{M_{0}} \vec{\varphi}_{0}, \vec{\varphi}_{0}\right\rangle_{M_{0}} \geq \Lambda_{M_{0}}\left\|\vec{\varphi}_{0}\right\|_{\left(\mathcal{H}^{0}\right)^{M_{0}}}^{2}-\operatorname{Re} \mathcal{C}_{M_{0}}\left(\vec{\varphi}_{0}, \vec{\varphi}_{0}\right) \quad$ for all $\vec{\varphi}_{0} \in\left(\mathcal{H}^{0}\right)^{M_{0}}$

Then, for $M=M_{0}+1$ we write

$$
\Theta=\left(\begin{array}{cc}
\Theta_{M_{0}} & 0 \\
0 & \vartheta
\end{array}\right), \quad \mathcal{A}=\left(\begin{array}{cc}
\mathcal{A}_{M_{0}} & 0 \\
\widetilde{A} & A
\end{array}\right), \quad \vec{\varphi}=\left(\begin{array}{c}
\vec{\varphi}_{0} \\
\varphi
\end{array}\right) .
$$

Here $\left.\widetilde{A}=\left(\left(\begin{array}{c}\delta_{M} \\ \delta_{j}\end{array}\right)\right) A_{\left(\delta_{M}-\vartheta_{j}\right)}\right)_{j=1, \ldots, M_{0}}^{\top}$ are the commutators in (4.5) for $m=M=$ $M_{0}+1$. Hence,

$$
\begin{aligned}
\operatorname{Re}\langle\Theta \mathcal{A} \vec{\varphi}, \vec{\varphi}\rangle \geq & \Lambda_{M_{0}}\left\|\vec{\varphi}_{0}\right\|_{\left(\mathcal{H}^{0}\right)^{M_{0}}}^{2}+\vartheta \Lambda_{1}\|\varphi\|_{\mathcal{H}^{0}}^{2} \\
& -\operatorname{Re} \mathcal{C}_{M_{0}}\left(\vec{\varphi}_{0}, \vec{\varphi}_{0}\right)-\operatorname{Re} \vartheta \mathcal{C}_{1}(\varphi, \varphi)-\vartheta K\left\|\vec{\varphi}_{0}\right\|_{\left(\mathcal{H}^{0}\right)^{M_{0}}}\|\varphi\|_{\mathcal{H}^{0}},
\end{aligned}
$$


where $K$ depends on the norms of $\widetilde{A}$ but is independent of $\vartheta>0$. Applying Cauchy's inequality yields

$$
-\vartheta K\left\|\vec{\varphi}_{0}\right\|_{\left(\mathcal{H}^{0}\right)^{M_{0}}}\|\varphi\|_{\mathcal{H}^{0}} \geq-\frac{1}{2} \Lambda_{M_{0}}\left\|\vec{\varphi}_{0}\right\|_{\left(\mathcal{H}^{0}\right)^{M_{0}}}^{2}-\frac{1}{2} \Lambda_{M_{0}}^{-1} \vartheta^{2} K^{2}\|\varphi\|_{\mathcal{H}^{0}}^{2} .
$$

Selecting $\vartheta=\Lambda_{1} \Lambda_{M_{0}} / K^{2}>0$ completes the proof.

If we apply the Galerkin method (4.1) to (4.6), i.e., we perform the bootstrapping algorithm for the evaluation of $\partial^{\alpha} \varphi$ in combination with the approximation procedure, then it is equivalent to: Find $\vec{\varphi}_{h} \in\left(\mathcal{H}_{h}\right)^{M}$ such that

$$
\left\langle\widetilde{\vec{\varphi}}_{h}, \mathcal{A} \vec{\varphi}_{h}\right\rangle=\left\langle\widetilde{\vec{\varphi}}_{h}, \vec{f}\right\rangle \quad \text { for all } \widetilde{\vec{\varphi}}_{h} \in\left(\mathcal{H}_{h}\right)^{M} .
$$

Because of (4.7), this method converges asymptotically of optimal order (4.4):

Proposition 4.2. For every $M$ and sufficiently small $h$, (4.8) admits unique solutions $\vec{\varphi}_{h}=\left(\varphi_{h}^{\left(\delta_{j}\right)}\right)_{j=1}^{M}$ which satisfy

$$
\left\|\partial^{\delta_{j}} \varphi-\varphi_{h}^{\left(\delta_{j}\right)}\right\|_{\mathcal{H}^{t}} \leq c(j) h^{\tau-t}\|\varphi\|_{\mathcal{H}^{\tau+\left|\delta_{j}\right|}} \quad \text { for } j=1, \ldots, M .
$$

Here $\tau$ and $t$ are as in (4.4).

4.2. Point value extraction for tangential derivatives. For the numerical evaluation of the Taylor expansion (1.7) we require normal derivatives of orders up to $K$ of the Cauchy data at $x_{0} \in \Gamma$. They can be expressed exclusively in terms of the tangential derivatives $\partial^{\delta_{j}} \varphi\left(x_{0}\right)$ of orders $\left|\delta_{j}\right| \leq K$ by Theorem 3.1. The tangential derivatives are approximated by $\varphi_{h}^{\left(\delta_{j}\right)}$ obtained from the Galerkin scheme (4.8).

Rather than evaluating $\varphi_{h}^{\left(\delta_{j}\right)}\left(x_{0}\right)$ directly from the Galerkin approximation $\varphi_{h}^{\left(\delta_{j}\right)}$, we calculate point values by averaging. We depart from the identity

$$
\left(-\Delta_{v}\right)^{k} G_{k}(r)=\delta(v-u)
$$

where

$$
G_{k}(r)=\frac{(-1)^{k}}{\pi 4^{k}((k-1) !)^{2}} r^{2(k-1)} \ln r^{2}
$$

is the fundamental solution of the polyharmonic operator $(-\Delta)^{k}, k=1,2, \ldots$, in $\mathbb{R}^{2}$ (see [36, p. 288]). This yields for any $f \in C_{0}^{\infty}\left(\mathbb{R}^{2}\right)$ the identities

$$
\begin{aligned}
f(u) & =(-1)^{k} \int_{\mathbb{R}^{2}}\left(\Delta_{v}^{k} f\right)(v) G_{k}(|v-u|) d v \\
& =(-1)^{k-1} \int_{\mathbb{R}^{2}} \nabla_{v}\left(\Delta_{v}^{k-1} f\right)(v) \cdot \nabla_{v} G_{k}(|v-u|) d v .
\end{aligned}
$$

Both relations can be written as

$$
f(u)=\int_{\mathbb{R}^{2}} \widetilde{\mathcal{P}}_{\ell}\left(\partial_{v}\right) f(v) \widetilde{G}_{\ell}(v-u) d v
$$

for any $\ell \in \mathbb{N}$, where $\widetilde{G}_{2 k}=G_{k}$ and $\widetilde{G}_{2 k-1}=\nabla_{v} G_{k}, k \in \mathbb{N}$, and where $\widetilde{\mathcal{P}}_{\ell}$ is the operator in the parametric plane defined in (4.12). We observe that $\widetilde{G}_{\ell}(z) \in$ 
$H_{\text {loc }}^{\ell-1-\varepsilon}\left(\mathbb{R}^{2}\right)$ for every $\varepsilon>0$. We will use formula (4.13) with $f(v)=\zeta(v-u) \partial^{\alpha} \varphi(v)$, where $\zeta \in C_{0}^{\infty}\left(\mathbb{R}^{2}\right)$ is a suitable cut-off function with $\zeta(0)=1$. With

$$
\widetilde{\mathcal{P}}_{\iota}\left(\partial_{v}\right)(\zeta(v-u) f(v))=\sum_{0 \leq|\beta| \leq \iota} d_{\beta}(v-u) \partial^{\beta} f(v)
$$

the extraction formula (4.13) takes the form

$$
\partial^{\alpha} \varphi(u)=\sum_{0 \leq|\beta| \leq \iota} \int_{\mathbb{R}^{2}} d_{\beta}(v-u)\left(\partial^{\alpha+\beta} \varphi(v)\right) \widetilde{G}_{\iota}(v-u) d v .
$$

Using here on the right-hand side the approximations $\varphi_{h}^{(\alpha+\beta)}$ obtained from the boot-strapping procedure (4.8), we recover the pointwise approximations

$$
\partial^{\alpha} \varphi(u) \sim \widehat{\varphi}_{h}^{(\alpha)}(u):=\sum_{0 \leq|\beta| \leq \iota} \int_{\mathbb{R}^{2}} d_{\beta}(v-u) \varphi_{h}^{(\alpha+\beta)}(v) \widetilde{G}_{\iota}(v-u) d v .
$$

For the corresponding error we have

Theorem 4.3. For the extracted derivatives $\widehat{\varphi}_{h}^{(\alpha)}$ in (4.16) we have the error estimate

$$
\left|\partial^{\alpha} \varphi(u)-\widehat{\varphi}_{h}^{(\alpha)}(u)\right| \leq c h^{\tau+t}\|\varphi\|_{\mathcal{H}^{\tau+|\alpha|+\iota}}
$$

where $\iota>\frac{\kappa}{2}+1$ and

$$
0 \leq t \leq \min \left\{d+\frac{\kappa}{2}, \iota-1-\frac{\kappa}{2}-\epsilon\right\}, 0 \leq \tau \leq \frac{\kappa}{2}+d,
$$

where $\kappa=0$ for equations of the second kind, $\kappa=1$ for equations of the first kind, and $\epsilon>0$ is arbitrary.

Note that the choice $\iota=d+2+\kappa$ ensures the maximum rate of convergence for the extracted derivatives in (4.16), whereas $\iota=d+1+\kappa$ implies an order $O\left(h^{2 d+1-\varepsilon}\right)$.

Proof of Theorem 4.3: Subtracting (4.15) from (4.16), we estimate

$$
\left|\partial^{\alpha} \varphi(u)-\widehat{\varphi}_{h}^{(\alpha)}(u)\right| \leq \sum_{0 \leq|\beta| \leq \iota}\left\|d_{\beta}\right\|_{L^{\infty}}\left\|\partial^{(\alpha+\beta)} \varphi-\varphi_{h}^{(\alpha+\beta)}\right\|_{H^{-t}(B+u)}\left\|\widetilde{G}_{\iota}\right\|_{H^{t}(B)} .
$$

Since $\widetilde{G}_{\iota} \in H_{\text {loc }}^{\iota-1-\epsilon}\left(\mathbb{R}^{2}\right)$, we must have $t \leq \iota-1-\epsilon$ for some $\epsilon>0$ for both types of integral equations. We discuss the cases now separately.

Case 1: Equation of the second kind. Here $\mathcal{H}^{t}=H^{t}$ in (4.9), i.e., (4.2) gives

$$
\left\|\partial^{(\alpha+\beta)} \varphi-\widehat{\varphi}_{h}^{(\alpha+\beta)}\right\|_{H^{-t}(B+u)} \leq c h^{\tau+t}\|\varphi\|_{H^{\tau+|\alpha|+|\beta|}}
$$

for $0 \leq t \leq d, 0 \leq \tau \leq d$, i.e., $0 \leq t \leq \min (d, \iota-1-\epsilon)$. This is (4.17) with $\kappa=0$.

Case 2: Equation of the first kind. The Galerkin solution $\varphi_{h}=\left(\sigma_{h}, \rho_{h}\right) \in \mathcal{H}_{h}$ satisfies, according to (4.2),

$$
\left\|\partial^{(\alpha+\beta)} \sigma-\widehat{\sigma}_{h}^{(\alpha+\beta)}\right\|_{H^{-t^{\prime}}(B+u)} \leq c h^{\tau^{\prime}+t^{\prime}}\left(\|\sigma\|_{H^{\tau^{\prime}+|\alpha|+|\beta|}}+\|\rho\|_{H^{\tau^{\prime}+|\alpha|+|\beta|+1}}\right),
$$

where $\frac{1}{2} \leq t^{\prime} \leq d+1,-\frac{1}{2} \leq \tau^{\prime} \leq d$, and

$$
\left\|\partial^{(\alpha+\beta)} \rho-\widehat{\rho}_{h}^{(\alpha+\beta)}\right\|_{H^{-t^{\prime \prime}}(B+u)} \leq c h^{\tau^{\prime \prime}+t^{\prime \prime}}\left(\|\rho\|_{H^{\tau^{\prime \prime}+|\alpha|+|\beta|}}+\|\sigma\|_{H^{\tau^{\prime \prime}+|\alpha|+|\beta|-1}}\right),
$$

where $-\frac{1}{2} \leq t^{\prime \prime} \leq d, \frac{1}{2} \leq \tau^{\prime \prime} \leq d+1$. Therefore

$$
\left\|\partial^{(\alpha+\beta)} \sigma-\widehat{\sigma}_{h}^{(\alpha+\beta)}\right\|_{H^{-t^{\prime}}(B+u)} \leq c h^{\tau^{\prime}+t^{\prime}}\|\varphi\|_{\mathcal{H}^{\tau^{\prime}+\frac{1}{2}+|\alpha|+|\beta|}}
$$




$$
\left\|\partial^{(\alpha+\beta)} \rho-\widehat{\rho}_{h}^{(\alpha+\beta)}\right\|_{H^{-t^{\prime \prime}}(B+u)} \leq \operatorname{ch}^{\tau^{\prime \prime}+t^{\prime \prime}}\|\varphi\|_{\mathcal{H}^{\tau^{\prime \prime}-\frac{1}{2}+|\alpha|+|\beta|}},
$$

where the indices must satisfy

$$
\begin{aligned}
& \frac{1}{2} \leq t^{\prime} \leq d+1, \quad-\frac{1}{2} \leq \tau^{\prime} \leq d, \quad t^{\prime} \leq \iota-1-\epsilon, \\
& -\frac{1}{2} \leq t^{\prime \prime} \leq d, \quad \frac{1}{2} \leq \tau^{\prime \prime} \leq d+1, \quad t^{\prime \prime} \leq \iota-1-\epsilon .
\end{aligned}
$$

Selecting here $\tau^{\prime}+\frac{1}{2}=\tau^{\prime \prime}-\frac{1}{2}=\tau$, we find that

$$
0 \leq \tau \leq d+\frac{1}{2}
$$

Selecting further $t^{\prime}-\frac{1}{2}=t^{\prime \prime}+\frac{1}{2}=t$, we find that $t$ must satisfy

$$
0 \leq t \leq d+\frac{1}{2}
$$

Moreover, we get from $G_{\iota} \in H^{\iota-1-\varepsilon}$ for any $\epsilon>0$ the conditions

$$
t^{\prime}=t+\frac{1}{2} \leq \iota-1-\epsilon \wedge t^{\prime \prime}=t-\frac{1}{2} \leq \iota-1-\epsilon,
$$

i.e.,

$$
0 \leq t \leq \min \left\{d+\frac{1}{2}, \iota-\frac{3}{2}-\epsilon\right\}
$$

where $\varepsilon>0$ is arbitrarily small, which is the assertion (4.17) with $\kappa=1$.

Remark 4.4 (on first kind equations). For $P=1$ we have $\mathcal{H}^{\tau}=H^{\tau-\frac{1}{2}}$ (weakly singular equations) and

$$
0 \leq \tau \leq d+\frac{1}{2} \text { and } 0 \leq t \leq \min \left\{d+\frac{1}{2}, \iota-\frac{3}{2}-\epsilon\right\} .
$$

For $P=0$ we have $\mathcal{H}^{\tau}=H^{\tau+\frac{1}{2}}$ (hypersingular equation) and

$$
0 \leq \tau \leq d+\frac{1}{2} \text { and } 0 \leq t \leq \min \left\{d+\frac{1}{2}, \iota-\frac{1}{2}-\epsilon\right\} .
$$

We see that the choices $\iota=d+2$ for the weakly singular and $\iota=d+1$ for the hypersingular equation and $\tau=d+\frac{1}{2}$ provides the convergence rate $h^{2 d+1-\epsilon}$ for the extracted data. This is almost optimal. The $\epsilon>0$ can be removed and the optimal rate $h^{2 d+1}$ achieved with the choices $\iota=d+3$ and $\iota=d+2$, respectively. In practice, this difference will hardly be noticable and may not be worth the extra term in (4.16) corresponding to $\iota=d+2$.

4.3. Error Estimates near $\Gamma$. We use Theorem 4.3 to analyze the error in the truncated Taylor expansion

$$
U(x)=\sum_{k=0}^{M}(-1)^{k} \frac{\epsilon^{k}}{k !}\left(\partial_{n}^{k} U\right)\left(x_{0}\right)+O\left(\epsilon^{M+1}\right)
$$

of the potential $U(x)$ at $x=x_{0}-\epsilon n\left(x_{0}\right)$, when replacing $\left(\partial_{n}^{k} U\right)\left(x_{0}\right)$ by the approximations $\left(\widehat{\partial_{n}^{k} U}\right)_{k}\left(x_{0}\right)$ obtained with the postprocessed tangential derivatives $\widehat{\varphi}_{h}^{(\alpha)}\left(x_{0}\right)$, i.e.,

$$
\widehat{U}_{h}^{M}(x):=\sum_{k=1}^{M}(-1)^{k} \frac{\epsilon^{k}}{k !} \widehat{\varphi}_{h}^{(\alpha)}\left(x_{0}\right) .
$$


Theorem 4.5. Assume that $\Gamma$ is smooth. For the approximate potential $\widehat{U}_{h}^{M}(x)$ at $x=x_{0}-\epsilon n\left(x_{0}\right)$, we have the error estimate

$\left|U(x)-\widehat{U}_{h}^{M}(x)\right| \leq C\left(h^{t+\tau}\|\varphi\|_{\mathcal{H}^{\tau+M+\iota}(\Gamma)}+\epsilon^{M+1}\left[\|\varphi\|_{H^{M+2+\delta}(\Gamma)}+\|\psi\|_{H^{M+2+\delta}(\Gamma)}\right]\right)$.

Here $\delta>0$ is arbitrary and $C$ depends on $\Omega, \delta, M, d$ and

$$
0 \leq \tau \leq d+\frac{\kappa}{2}, 0 \leq t \leq \min \left\{d+\frac{\kappa}{2}, \iota-1-\frac{\kappa}{2}-\delta\right\},
$$

with $\kappa$ as in Theorem 4.3.

Proof. Inspecting the proof of Theorem 3.1, we get with (2.24)

$$
\begin{aligned}
\partial_{h}^{k} U\left(x_{0}\right) & =\mathcal{P}_{1, k}(\partial)\left(\gamma_{0} U\right)\left(x_{0}\right)+\mathcal{P}_{2, k-1}(\partial)\left(\gamma_{1} U\right)\left(x_{0}\right) \\
& =\mathcal{P}_{1, k}(\partial)[P \psi+(I-P) \varphi]+\mathcal{P}_{2, k-1}[(I-P) \psi+P \varphi] \\
& =\sum_{|\alpha| \leq k} c_{\alpha}^{1}\left(\partial^{\alpha} \varphi\right)\left(x_{0}\right)+\sum_{|\alpha| \leq k} c_{\alpha}^{2}\left(\partial^{\alpha} \psi\right)\left(x_{0}\right) .
\end{aligned}
$$

Since $\psi$ is given explicitly, we have with Theorem 4.3 the error estimate

$$
\begin{aligned}
\left|\partial_{h}^{k} U(x)-\left(\widehat{\partial_{h}^{k} U}\right)_{h}\left(x_{0}\right)\right| & =\left|\sum_{|\alpha| \leq k} c_{\alpha}^{1}\left[\left(\partial^{\alpha} \varphi\right)\left(x_{0}\right)-\widehat{\varphi}_{h}^{(\alpha)}\left(x_{0}\right)\right]\right| \\
& \leq C \cdot \sum_{|\alpha| \leq k}\left|\left(\partial^{\alpha} \varphi\right)\left(x_{0}\right)-\widehat{\varphi}_{h}^{(\alpha)}\left(x_{0}\right)\right| \\
& \leq c h^{t+\tau} \sum_{|\alpha| \leq k}\|\varphi\|_{\mathcal{H}^{\tau+|\alpha|+\iota}} \leq c h^{t+\tau}\|\varphi\|_{\mathcal{H}^{\tau+k+\iota}} .
\end{aligned}
$$

Using the Taylor formula

$$
U(x)=\sum_{k=0}^{M}(-1)^{k} \frac{\epsilon^{k}}{k !}\left(\partial_{n}^{k} U\right)\left(x_{0}\right)+O\left(\epsilon^{M+1}\right)
$$

and the corresponding approximation

$$
\widehat{U}_{h}^{M}(x):=\sum_{k=0}^{M}(-1)^{k} \frac{\epsilon^{k}}{k !} \widehat{\left(\partial_{n}^{k} U\right)}\left(x_{0}\right),
$$

we find the error estimate

$$
\begin{aligned}
\left|U(x)-\widehat{U}_{h}^{M}(x)\right| & \leq \sum_{k=0}^{M} \frac{\epsilon^{k}}{k !}\left|\partial_{n}^{k} U\left(x_{0}\right)-\widehat{\left(\partial_{n}^{k} U\right)}\left(x_{0}\right)\right|+O\left(\epsilon^{M+1}\right) \\
& \leq c \sum_{k=0}^{M} \sum_{|\alpha| \leq k}^{M}\left|\left(\partial^{\alpha} \varphi\right)\left(x_{0}\right)-\widehat{\varphi}_{h}^{(\alpha)}\left(x_{0}\right)\right|+O\left(\epsilon^{M+1}\right) \\
& \leq c h^{t+\tau}\|\varphi\|_{\mathcal{H}^{\tau+M+\iota}}+O\left(\epsilon^{M+1}\right) .
\end{aligned}
$$

The remainder $O\left(\epsilon^{M+1}\right)$ is equal to

$$
C_{M} \epsilon^{M+1}\left(\partial_{n}^{M+1} U\right)(\zeta), \quad \zeta \in\left(x_{0}, x_{0}-\epsilon n\left(x_{0}\right)\right) .
$$


Using the embedding theorem, we get, using $\mathcal{L} U=0$ in $\Omega$ and the smoothness of $\Gamma$, that

$$
\begin{aligned}
\left|\left(\partial_{n}^{M+1} U\right)(\zeta)\right| & \leq c_{\delta}(\Omega)\|U\|_{H^{M+1+\frac{3}{2}+\delta}(\Omega)} \\
& \leq c_{\delta}(\Omega)\left\|\gamma_{0} U\right\|_{H^{M+2+\delta}(\Gamma)} \\
& \leq c_{\delta}(\Omega)\|P \psi+(I-P) \varphi\|_{H^{M+2+\delta}(\Gamma)} \\
& \leq c_{\delta}(\Omega)\left(\|\psi\|_{H^{M+2+\delta}(\Gamma)}+\|\varphi\|_{H^{M+2+\delta}(\Gamma)}\right)
\end{aligned}
$$

which completes the proof.

Remark 4.6. In the previous theorem, we assumed that $\Gamma$ is smooth. The result holds, however, also in the case of piecewise smooth $\Gamma$, if $x_{0}$ is sufficiently far away from the set $S$ of edges and vertices.

\section{A NUMERICAL EXAMPLE}

The following two-dimensional example is due to H. Schulz and can be found in more detail in [32]. Consider the interior Dirichlet problem with the Laplacian,

$$
\Delta U=0 \quad \text { in } \Omega=\left\{x \mid x_{1}^{2}+4 x_{2}^{2}<0.36\right\}, \quad U_{\left.\right|_{\Gamma}}=\psi=\log |x-y|,
$$

where $\Gamma: \chi(t)=(0.6 \cos t, 0.3 \sin t), t \in[0,2 \pi]$, and $y=y_{0}+\delta n\left(y_{0}\right), y_{0}=\chi(3 \pi / 4)$, $\delta=0.4$.

The boundary integral equations of the first kind for $\varphi=\frac{\partial U}{\partial n} \mid \Gamma$ and its tangential derivative $\varphi^{\prime}:=\frac{d \varphi}{d s}=\dot{\varphi}|\dot{\chi}|^{-1}$, where $d / d t=\stackrel{\bullet}{ }$, read

$$
\begin{aligned}
A \varphi & :=-\frac{1}{2 \pi} \int_{0}^{2 \pi} \ln |x-\chi(t)| \varphi(t)|\dot{\chi}| d t \\
& =f(t):=0.3 \cos t+\frac{1}{2 \pi} \int_{0}^{2 \pi} \frac{n(t) \cdot(\chi(t)-x)}{|\chi(t)-x|^{2}} 0.3 \cos t|\dot{\chi}| d t
\end{aligned}
$$

and

$$
A \varphi^{\prime}=-\frac{1}{2 \pi} \int_{0}^{2 \pi} \ln |x-\chi(t)| \dot{\varphi}(t) d t=\dot{f}|\dot{\chi}|^{-1}-V_{(1)} \varphi
$$

with $V_{(1)}$ given by (3.38). The system (5.2), (5.3) is numerically solved by Galerkin's method on the family of regular partitions and grids with $h=2 \pi / N$ for $N=2^{\ell}$ with $\ell=4, \ldots, 8$ and with piecewise constant periodic functions $S_{h}^{1,0}([0,2 \pi])$. Table 1 shows the $L^{2}$-error of $\varphi_{h}, \varphi_{h}^{(1)}$ and of the recovered $\widehat{\varphi}_{h}$ (with $\iota=1$ ) for successively refined uniform meshes, together with the convergence rates $\alpha$. In Figure 3 we see the different behaviour of the pointwise errors of a): direct evaluation of the representation formula

$$
U_{h}(x):=-\frac{1}{2 \pi} \int_{0}^{2 \pi} \ln |x-\chi(t)| \varphi_{h}|\dot{\chi}| d t+\frac{1}{4 \pi} \int_{0}^{2 \pi} \frac{n(t) \cdot(\chi(t)-x)}{|\chi(t)-x|^{2}} x_{1}(t)|\dot{\chi}| d t
$$


TABLE 1. $L^{2}$-errors of $\varphi_{h}, \varphi_{h}^{(1)}, \widehat{\varphi}_{h}$.

\begin{tabular}{|r|c|c|c|c|c|c|}
\hline$N$ & $\left\|\varphi-\varphi_{h}\right\|_{L^{2}(\Gamma)}$ & $\alpha$ & $\left\|\partial_{s} \varphi-\varphi_{h}^{(1)}\right\|_{L^{2}(\Gamma)}$ & $\alpha$ & $\left\|\varphi-\hat{\varphi}_{h}\right\|_{L^{2}(\Gamma)}$ & $\alpha$ \\
\hline 8 & 0.724432 & & 0.634889 & & 0.162597 & \\
16 & 0.338135 & 1.1 & 0.833876 & 0.5 & 0.032748 & 2.3 \\
32 & 0.167685 & 1.0 & 0.941592 & 0.9 & 0.006806 & 2.2 \\
64 & 0.083584 & 1.0 & 0.468299 & 1.0 & 0.001583 & 2.1 \\
128 & 0.041751 & 1.0 & 0.233676 & 1.0 & 0.000389 & 2.0 \\
256 & 0.020869 & 1.0 & 0.116770 & 1.0 & 0.000097 & 2.0 \\
\hline
\end{tabular}

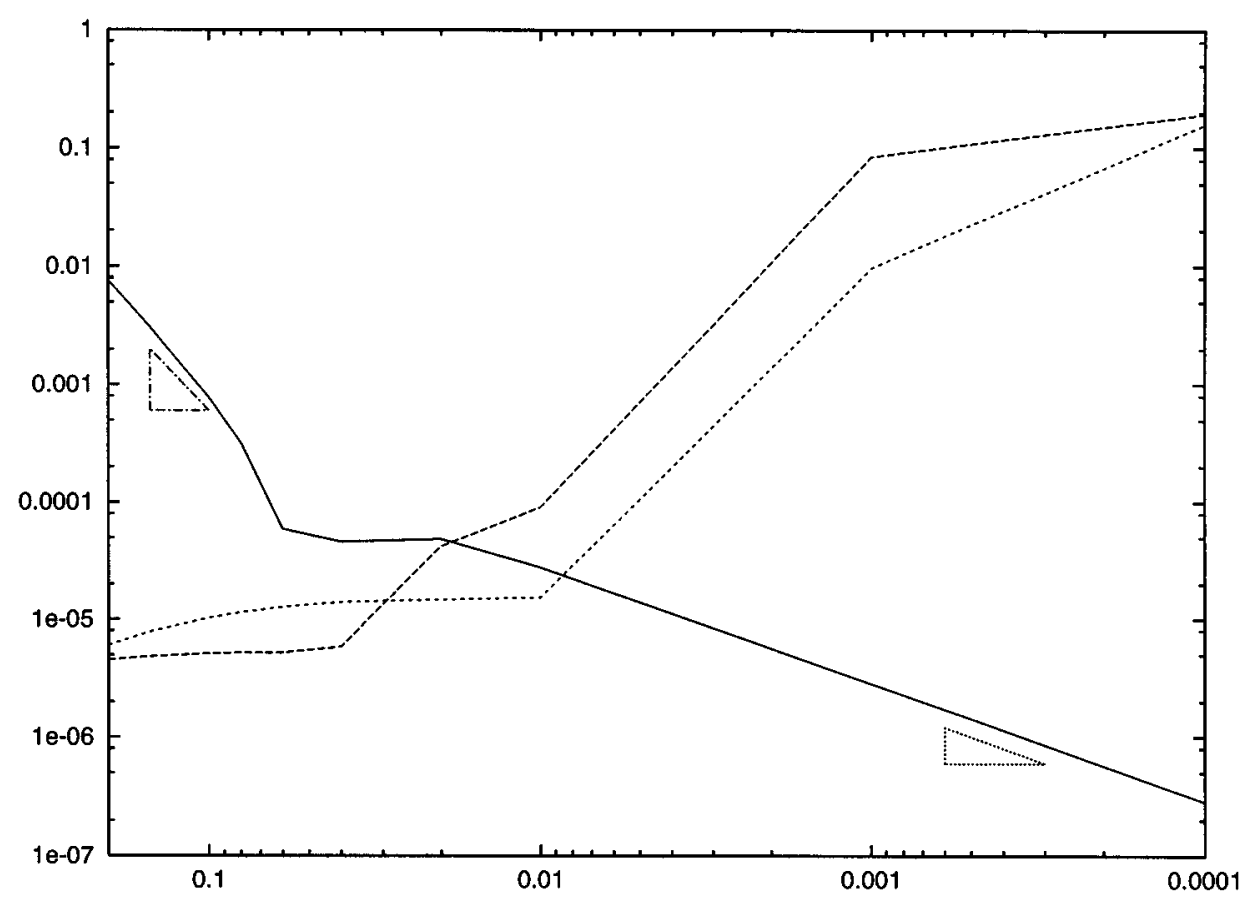

FiguRE 3. Errors of $U_{h}(x)$ and $\widehat{U}_{h}(x)$ at $x=(0.6-\epsilon, 0)$ versus $\epsilon$

with various numbers of quadrature points (broken lines) and b): of the Taylor approximation

$$
\hat{U}_{h}(x)=\psi\left(x_{0}\right)-\epsilon \hat{\varphi}_{h}\left(x_{0}\right)+\epsilon^{2}\left(\kappa \hat{\varphi}_{h}\left(x_{0}\right)-\ddot{\psi}\left(x_{0}\right)\right)
$$

for points $x=(0.6-\epsilon, 0)$ with $\epsilon \in\left[10^{-8}, 10^{-1}\right]$ and $x_{0}=(0.6,0)$ where $N=64$. Here $\kappa$ is the curvature of $\Gamma$.

The solid line in Figure 3 shows that the error $\left|\hat{U}_{h}\left(x_{0}-\epsilon n\left(x_{0}\right)\right)-U\left(x_{0}-\epsilon n\left(x_{0}\right)\right)\right|$ exhibits two different asymptotic behaviours - namely $O\left(\epsilon^{3}\right)$ first and, for small $\epsilon, O(\epsilon)$. From the Taylor expansion

$$
U(x)=\psi\left(x_{0}\right)-\epsilon \varphi\left(x_{0}\right)+\epsilon^{2}(\kappa \varphi-\ddot{\psi})\left(x_{0}\right)+O\left(\epsilon^{3}\right)
$$

we expect $O\left(\epsilon^{3}\right)$ behaviour. Note, however, that the computed approximation (5.5) uses the extracted values $\hat{\varphi}_{h}\left(x_{0}\right)$ rather than $\varphi\left(x_{0}\right)$. For small $\epsilon$, therefore, the error 
$\left|\varphi\left(x_{0}\right)-\hat{\varphi}_{h}\left(x_{0}\right)\right| \epsilon$ dominates, as we see in Figure 3. The crossover point from $O\left(\epsilon^{3}\right)$ to $O(\epsilon)$ behaviour is determined by the accuracy of $\hat{\varphi}_{h}\left(x_{0}\right)$, the superconvergent, extracted point value of the Cauchy datum $\varphi$. We also note that the approximations $U_{h}(x)$ obtained by quadrature evaluation of the representation formula (5.4) are clearly inferior at points close to $\Gamma$, even if many quadrature points are used.

\section{Appendix. The Representation of SECOND order Elliptic Systems IN TUbular COORDinates [17, Chapter 3$]$}

For the local surface representation of $\Gamma_{j} \subset \mathbb{R}^{3}$, we have in addition to (1.8) (1.12) the well-known Weingarten formulae for the curvatures (see e.g., [24, Chap. III, 11.7]):

$$
\begin{gathered}
L_{\lambda \nu}:=\chi_{\mid \lambda \nu} \cdot n, \quad L_{\lambda}^{\mu}:=\sum_{\nu=1}^{2} L_{\lambda \nu} \gamma^{\nu \mu} \text { for } \lambda, \mu=1,2 \\
n_{\mid \lambda}=-\sum_{\mu=1}^{2} L_{\lambda}^{\mu} \chi_{\mid \mu}, K=\operatorname{det}\left(\left(L_{\lambda}^{\mu}\right)\right), 2 H L_{\nu \mu}-K \gamma_{\nu \mu}=\sum_{\lambda=1}^{2} L_{\nu \lambda} K_{\mu}^{\lambda} .
\end{gathered}
$$

For the diffeomorphism defined in (1.13), we have the following representations of the Riemann fundamental tensor:

$$
\begin{gathered}
g_{j k}:=\frac{\partial y}{\partial v_{j}} \cdot \frac{\partial y}{\partial v_{k}} \quad \text { for } j, k=1,2,3 ; \\
g_{\nu \lambda}=\left(1-v_{3}^{2} K\right) \gamma_{\nu \lambda}-2 v_{3}\left(1-v_{3} H\right) L_{\nu \lambda} \quad \text { for } \lambda, \nu=1,2 ; \\
g_{3 \lambda}=0 \text { for } \lambda=1,2 \text { and } g_{33}=1 .
\end{gathered}
$$

If $\Phi$ is differentiable, then

$$
\frac{\partial(\Phi \circ y(v))}{\partial v_{3}}=\partial_{n} \Phi=\nabla \Phi \cdot n \quad \text { in } \mathcal{U} .
$$

The Christoffel symbols

$$
G_{j k}^{r}:=\frac{\partial^{2} y}{\partial v_{j} \partial v_{k}} \cdot \sum_{\ell=1}^{3} \frac{\partial y}{\partial v_{\ell}} g^{\ell r}, \quad \text { where }\left(\left(g^{\ell r}\right)\right)=\left(\left(g_{j k}\right)\right)^{-1},
$$

satisfy in $\mathcal{U}$ :

$$
\begin{aligned}
G_{\nu \mu}^{3} & =\left(1-2 v_{3} H\right) L_{\nu \mu}+v_{3} K \gamma_{\nu \mu} \quad \text { and } \quad G_{3 \lambda}^{3}=G_{\lambda 3}^{3}=0 \quad \text { for } \nu, \mu, \lambda=1,2 ; \\
\left(\begin{array}{c}
G_{3 \lambda}^{1} \\
G_{3 \lambda}^{2}
\end{array}\right) & =-\left(\begin{array}{c}
L_{\lambda}^{1} \\
L_{\lambda}^{2}
\end{array}\right)-\sum_{\ell=1}^{\infty} v_{3}^{\ell}\left(\begin{array}{c}
L_{1}^{1} L_{2}^{1} \\
L_{1}^{2} L_{2}^{2}
\end{array}\right)^{\ell}\left(\begin{array}{c}
L_{\lambda}^{1} \\
L_{\lambda}^{2}
\end{array}\right) ; \\
G_{\lambda \mu}^{\varrho} & =\sum_{\alpha=1}^{2}\left(\chi_{\mid \alpha}-v_{3} \sum_{\beta=1}^{2} L_{\alpha}^{\beta} \chi_{\mid \beta}\right) \cdot\left(\begin{array}{c}
\left.\chi_{\mid \lambda \mu}-v_{3} \sum_{\beta=1}^{2} L_{\lambda}^{\beta} \chi_{\mid \beta \mu}\right) g^{\alpha \varrho} \\
\text { for } \lambda, \mu, \varrho=1,2 ; \\
G_{33}^{r}
\end{array}=0 \quad \text { for } r=1,2,3 .\right.
\end{aligned}
$$


The local representation of the operator $\mathcal{L} U$ reads

$$
\mathcal{L} U \equiv \sum_{j, k} \frac{\partial}{\partial x_{j}}\left(a_{j k}(x) \frac{\partial U}{\partial x_{k}}\right)+c U=\left\{\mathcal{P}_{2}+\mathcal{P}_{1} \frac{\partial}{\partial n}+\mathcal{P}_{0}\left(\frac{\partial}{\partial n}\right)^{2}\right\} U
$$

where, in $\mathcal{U}$,

$$
\begin{aligned}
& \mathcal{P}_{0}=\sum_{j, k=1}^{3} n_{j} a_{j k} n_{k} \\
& \mathcal{P}_{1}=\sum_{\lambda=1}^{2}\left(\sum_{j, k=1}^{3} a_{j k} \sum_{\nu=1}^{2} y_{j \mid \nu} n_{k} g^{\nu \lambda}\right) \frac{\partial}{\partial v_{\lambda}} \\
& +\left(2 \frac{\sqrt{\gamma}}{\sqrt{g}}\left(v_{3} K-H\right)-\sum_{\kappa=1}^{2} G_{\kappa 3}^{\kappa}\right) \sum_{j, k=1}^{3} n_{j} a_{j k} n_{k} \\
& -\sum_{j, k=1}^{3} \sum_{\varrho, \lambda, \nu=1}^{2} a_{j k} y_{j \mid \varrho} L_{\lambda}^{\nu} \chi_{k \mid \nu} g^{\varrho \lambda} \\
& +\sum_{j, k=1}^{3} \frac{\partial a_{j k}}{\partial v_{3}} n_{j} n_{k}+\sum_{j, k, \ell=1}^{3} \sum_{\varrho, \nu=1}^{2} \frac{\partial a_{j k}}{\partial x_{\ell}} y_{\ell \mid \varrho} y_{j \mid \nu} n_{k} g^{\varrho \nu} \\
& \mathcal{P}_{2}=\sum_{\kappa, \lambda=1}^{2} \frac{1}{\sqrt{g}} \frac{\partial}{\partial v_{\kappa}}\left\{\sqrt{g} \sum_{j, k=1}^{3} \sum_{\mu, \nu=1}^{2} a_{j k} y_{j \mid \nu} y_{k \mid \mu} g^{\nu \kappa} g^{\mu \lambda}\right\} \frac{\partial}{\partial v_{\lambda}} \\
& +\sum_{\lambda=1}^{2}\left\{\sum _ { j , k = 1 } ^ { 3 } a _ { j k } n _ { j } \left(\sum _ { \mu = 1 } ^ { 2 } \left[\frac{2 \sqrt{\gamma}}{\sqrt{g}}\left(v_{3} K-H\right) y_{k \mid \mu}\right.\right.\right. \\
& \left.\left.-\sum_{\nu=1}^{2}\left(\chi_{k \mid \nu} L_{\mu}^{\nu}+y_{k \mid \nu} G_{\mu 3}^{\nu}\right)\right] g^{\mu \lambda}-\sum_{\mu, \nu=1}^{2} y_{k \mid \nu} g^{\mu \nu} G_{\mu 3}^{\lambda}\right) \\
& \left.+\sum_{\mu=1}^{2}\left(\sum_{j, k=1}^{3} \frac{\partial a_{j k}}{\partial v_{3}} n_{j}\right) g^{\mu \nu}\right\} \frac{\partial}{\partial v_{\lambda}}+c .
\end{aligned}
$$

\section{ACKNOWLEDGMENTS}

The work of C. Schwab was partially supported by the DFG priority research programme "Randelementmethoden" and the Sonderforschungsbereich 404 in Stuttgart within its guest-programme; the work of W.L. Wendland was partially supported by the ETH Zürich during his visit there.

The authors want to express their gratitude to H. Schulz, who programmed and analyzed the numerical example in Section 5.

\section{REFERENCES}

[1] I. Babuška and A.K. Aziz: Survey lectures on the mathematical foundations of the finite element method. In: The Mathematical Foundation of the Finite Element Method with Applications to Partial Differential Equations (ed. A. K. Aziz), Academic Press, New York (1972) 3-359. MR 54:9111 
[2] Yu.D. Burago and V.G. Maz'ja: Potential Theory and Function Theory for Irregular Regions. Seminars in Math., V.A. Steklov Math. Inst., Leningrad, Vol. 3 (1967), English Transl. Consultants Bureau, New York (1969). MR 37:3031; MR 39:1633

[3] M. Costabel: Starke Elliptizität von Randintegraloperatoren erster Art, Habilitationsschrift Technische Hochschule Darmstadt, Fachbereich Mathematik, Preprint No. 868 (1984).

[4] M. Costabel: Boundary integral operators on Lipschitz domains - elementary results, SIAM J. Math. Anal. 19 (1987) 613-626. MR 89h:35090

[5] M. Costabel and M.Dauge: On representation formulas and radiation conditions. Math. Methods Appl. Sci. 20 (1997), 133-150. MR 97m:35031

[6] M. Costabel and W. L. Wendland: Strong ellipticity of boundary integral operators, Journ. Reine Angew. Math. 372 (1986) 34-63. MR 88c:35048

[7] T.A. Cruse and J.D. Richardson: Continuity of the elastic BIE formulation, (Preprint Dept. Mech. Engrg. Vanderbilt Univ. 1995).

[8] T.A. Cruse and Q. Huang: On the nonsingular traction-BIE in elasticity. Intern. J. Numer. Methods Engrg. 37 (1994), 2041-2072. MR 95a:73074

[9] R. Dautray and J.-L. Lions: Mathematical Analysis and Numerical Methods for Science and Technology, Vol. 4: Integral Equations and Numerical Methods. Springer-Verlag, Berlin 1990. MR 91h:00004b

[10] C. Fiedler and L.Gaul: Limiting procedures and calculation of boundary stresses in threedimenional boundary element method. In: Advances in Comp. Mechanics (M. Papadrakakis and B.H.V. Topping, eds.), Civil-Comp. Ltd., Edinburgh (1994), 311-322.

[11] M. Guiggiani: "Accurate evaluation of stresses on the boundary using hypersingular integral equations" in: Proc. of the First European Conference on Numerical Methods in Engineering, Brussels, 7-11 Sept. 1992. Ch. Hirsch (Ed.), Elsevier Science Publishers.

[12] M. Guiggiani, G. Krishnasamy, T. J. Rudolphi and F. J. Rizzo: A general algorithm for the numerical solution of hypersingular boundary integral equations, Trans. ASME J. Appl. Mech. 59 (1992), 604-614. MR 93f:73109

[13] F. Hartmann: Elastic potentials on piecewise smooth surfaces, J. Elasticity 12 (1982) 31-50. MR 83f:73025b

[14] G. C. Hsiao and W. L. Wendland: A finite element method for some integral equations of the first kind, J. Math. Anal. Appl. 58 (1977) 449-481. MR 57:1945

[15] G. C. Hsiao and W. L. Wendland: The Aubin-Nitsche lemma for integral equations, J. Integral Equations 3 (1981) 299-315. MR 83j:45019

[16] G. C. Hsiao and W. L. Wendland: On a boundary integral method for some exterior problems in elasticity, Trudy Tbiliss. Univ. Mat. Mekh. Astr. 18 (1985), 31-60. MR 88f:73019

[17] G. C. Hsiao and W. L. Wendland: Variational Methods for Boundary Integral Equations. In preparation.

[18] O. Huber, A. Lang and G. Kuhn: Evaluation of the stress tensor in 3D elastostatics by direct solving of hypersingular integrals. Comp. Mechanics 12 (1993) 39-50. MR 94e:73065

[19] F. John: Plane Waves and Spherical Means Applied to Partial Differential Equations, Wiley Interscience, New York 1955. MR 17:746d

[20] J.H. Kane and C. Balakrishna: Symmetric Galerkin boundary formulations employing curved elements. Intern. J. Numer. Methods Engrg. 36 (1993) 2157-2187. MR 94c:73067

[21] R. Kieser: Hypersinguläre Operatoren und einseitige Sprungrelationen in der Methode der Randelemente, Doctoral Dissertation, Stuttgart University 1991.

[22] R. Kieser, C. Schwab and W. L. Wendland: Numerical evaluation of singular and finite part integrals on curved surfaces using symbolic manipulation, Computing 49 (1992), 279-301. MR 93i: 76037

[23] J.C. Lachat and J.O. Watson: Effective numerical treatment of boundary integral equations: A formulation for three-dimensional elastostatics. Intern. J. Numer. Methods Engrg. 10 (1979) 991-1005.

[24] D. Laugwitz: Differentialgeometrie. B.G. Teubner, Stuttgart, 1960. MR 22:7061

[25] J. L. Lions and E. Magenes: Non-Homogeneous Boundary Value Problems and Applications I, Springer-Verlag, Berlin, 1972. MR 50:2670

[26] T. Matsumoto and M. Tanaka: Boundary stress calculation using regularized boundary integral equation for displacement gradients. Intern. J. Numer. Methods Engrg. 36 (1993) $783-797$. 
[27] S.G. Mikhlin: Multidimensional Singular Integrals and Integral Equations. Pergamon Press, Oxford 1965. MR 32:2866

[28] C. Miranda: Partial Differential Equations of Elliptic Type. Springer-Verlag, Berlin 1970. MR 44:1924

[29] C. B. Morrey, Jr.: Multiple Integrals in the Calculus of Variations, Springer-Verlag, Berlin, 1966. MR 34:2380

[30] J. Nečas: Les Méthodes Directes en Théorie des Equations Elliptiques, Masson, Paris 1967. MR 37:3168

[31] J.C. Nedelec and J. Planchard: Une méthode variationelle d'élements finis pour la résolution numerique d'un problème exterieur dans $\mathbb{R}^{3}$, Revue Franc. Automatique Inf. Rech. Operationelle 3 (1973) 105-129. MR 54:11992

[32] H. Schulz, Ch. Schwab and W.L. Wendland: An extraction technique for BEM. In: Lecture Notes on Numerical Fluid Mechanics, Vol. 54 (W. Hackbusch \& G. Wittum, eds.), Vieweg Verlag (1996), pp. 219-231.

[33] C. Schwab: Variable order composite quadrature of singular and nearly singular integrals. Computing 53 (1994) 173-194. MR 96a:65035

[34] C. Schwab and W. L. Wendland: Kernel properties and representations of boundary integral operators, Mathematische Nachrichten 156 (1992) 187-218. MR 94g:65135

[35] C. Schwab and W. L. Wendland: On numerical cubatures of singular surface integrals in boundary element methods, Numerische Mathematik 62 (1992) 343-369. MR 93h:65035

[36] L. Schwartz: Théorie des Distributions. Hermann, Paris 1966 (3rd. ed.). MR 35:730

[37] F. Treves: Pseudodifferential and Fourier Integral Operators. Vol. 1. Plenum Press, New York 1980. MR 82i: 35173

[38] B.R. Vainberg: Asymptotic Methods in Equations of Mathematical Physics. Gordon \& Breach, New York 1989. MR 91h:35081

[39] W.L. Wendland: Die Behandlung von Randwertaufgaben im $\mathbb{R}^{3}$ mit Hilfe von Einfach-und Doppelschichtpotentialen. Numer. Math. 11 (1968) 380-404. MR 37:7103

[40] W.L. Wendland: Strongly elliptic boundary integral equations. In: The State of the Art in Numerical Analysis (eds. A. Iserles, M. Powell), Clarendon Press, Oxford (1987) 511-561. MR 88m:65209

Seminar für Angewandte Mathematik, ETH ZÜrich, CH-8092 Zürich, Switzerland

E-mail address: schwab@sam.math.ethz.ch

Mathematisches Institut A, Universität Stuttgart, Pfaffenwaldring 57, D-70569 Stuttgart, Germany

E-mail address: wendland@mathematik.uni-stuttgart.de 